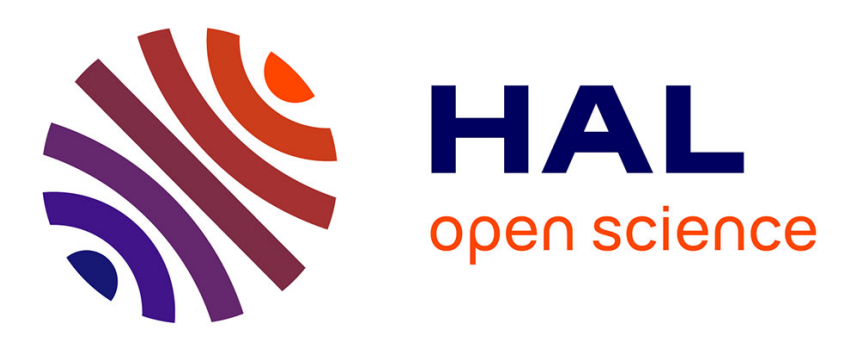

\title{
Successive enlargement of filtrations and application to insider information *
}

Christophette Blanchet-Scalliet, Caroline Hillairet, Ying Jiao

\section{To cite this version:}

Christophette Blanchet-Scalliet, Caroline Hillairet, Ying Jiao. Successive enlargement of filtrations and application to insider information *. Advances in Applied Probability, 2017, 49 (3), https://doi.org/10.1017/apr.2017.17. hal-01259711

\section{HAL Id: hal-01259711 \\ https://hal.science/hal-01259711}

Submitted on 20 Jan 2016

HAL is a multi-disciplinary open access archive for the deposit and dissemination of scientific research documents, whether they are published or not. The documents may come from teaching and research institutions in France or abroad, or from public or private research centers.
L'archive ouverte pluridisciplinaire HAL, est destinée au dépôt et à la diffusion de documents scientifiques de niveau recherche, publiés ou non, émanant des établissements d'enseignement et de recherche français ou étrangers, des laboratoires publics ou privés. 


\title{
Successive enlargement of filtrations and application to insider information*
}

\author{
Christophette Blanchet-Scalliet ${ }^{\dagger}$ Caroline Hillairet ${ }^{\ddagger}$ Ying Jiao $^{\S}$
}

January 20, 2016

\begin{abstract}
We model in a dynamic way an insider's private information flow which is successively augmented by a family of initial enlargement of filtrations. According to the a priori available information, we propose several density hypotheses which are presented in hierarchical order from the weakest one to the stronger ones. We compare these hypotheses, in particular, with the Jacod's one, and deduce conditional expectations under each of them by providing consistent expressions with respect to the common reference filtration. Finally, this framework is applied to a default model with insider information on the default threshold and some numerical illustrations are performed.
\end{abstract}

Keywords: enlargement of filtrations, density hypothesis, insider information.

AMS classification: $60 \mathrm{G}$; $91 \mathrm{G} 40$.

\section{Introduction}

Modeling information is a crucial subject in financial markets. The mathematical tool is based on the theory of initial enlargement of filtration by a random variable, which has been developed by the French school in the 70's-80's by Jacod [17, 18, Jeulin [19], Jeulin and Yor [21, etc. This theory receives a new focus in the 90's for its application in finance notably for problems

${ }^{*}$ We thank Peter Imkeller, Monique Jeanblanc, Philip Protter and Shiqi Song for interesting and helpful discussions and comments.

${ }^{\dagger}$ Université de Lyon - CNRS, UMR 5208, Institut Camille Jordan - Ecole Centrale de Lyon, 36 avenue Guy de Collongue, 69134 Ecully Cedex, FRANCE. Email:christophette.blanchet@ec-lyon.fr

${ }^{\ddagger}$ Ensae ParisTech, CREST- Email: caroline.hillairet@ensae.fr. The author acknowledges funding from the research programs Chaire Risques Financiers of Fondation du Risque, Chaire Marchés en mutation of the Fédération Bancaire Française and Chaire Finance et développement durable of EDF and Calyon.

${ }^{\S}$ Université Claude Bernard - Lyon 1, Institut de Science Financière et d'Assurances, 50 Avenue Tony Garnier, 69007, Lyon France. Email: ying.jiao@univ-lyon1.fr 
occurring in insider modeling. When there is an insider, her information is often modeled by the enlargement of the common information filtration by the insider's private information and we investigate problems such as the existence of arbitrage or the value of private information, see e.g. Grorud and Pontier [12], Amendinger, Imkeller and Schweizer [2] and Imkeller [16]. Classically in these above papers, the extra information $L$ is revealed at the initial time but does not evolve or get more accurate through time.

In this present paper, our aim is to generalize previous works and consider an insider who can adjust her extra information with time. Let $t_{i},(i=1, \ldots, n)$ be a family of discrete times and $L^{i}$ be random variables modeling the extra information available at time $t_{i}$. The insider's information, which is modeled by the filtration $\mathbb{G}^{I}$, is given by the successive initial enlargement at time $t_{i}$ by the random variable $L^{i}$. In [12] and [1, Jacod's hypothesis or the so-called density hypothesis, which assumes the equivalence between the conditional law of $L$ with respect to the common reference filtration and the law of $L$, plays an important role. It implies in particular the existence of an equivalent martingale measure and thus No Free Lunch with Vanishing Risk. Moreover, following Föllmer and Imkeller [11, it has been constructed in [12] an equivalent martingale measure under which the reference filtration is independent to the random variable $L$. Our methodology consists of generalizing these properties in the framework of successive initial enlargement. We propose several density hypotheses in a hierarchical order. We show that if a density hypothesis is supposed at each step between the conditional laws of $L^{i}$ with respect to the previous information at different times, we obtain families of probability measures with nice properties. Indeed, under this successive density hypothesis, we construct a family of probabilities $\mathbb{P}^{i}, i=1, \ldots, n$ which decouple at time $t_{i}$ the random variable $L^{i}$ and $\mathcal{G}_{t_{i}^{-}}^{I}$ the available information up to time $t_{i}^{-}$. However, this first family obtained by a natural induction does not preserve at time $t_{i}$ the law of the next random variables $L^{k}, i<k \leq n$. To overcome this inconvenience, we propose a second family of probability measures $\mathbb{Q}^{i}, i=1, \ldots, n$ constructed by a backward change of probability measure. Then, we focus on conditional expectation with successive information. The use of the family $\mathbb{Q}^{i}$ allows to obtain an evaluation formula in terms of $\mathbb{F}$-conditional expectations where $\mathbb{F}$ is the common reference information. Our approach, although less general than the local method solution approach introduced by Song [26, 27, provides nevertheless tractable formulas in particular for the computation of conditional expectation, which are useful for financial applications. From this successive density hypothesis, we derive in addition stronger formulations where the a priori available information concerns the non-trivial or trivial initial $\sigma$-algebra, which are more similar to the classical density hypothesis of Jacod in initial enlargement framework. Moreover, another point of view is to consider a global initial enlargement of the reference filtration $\mathbb{F}$ by the random vector $\boldsymbol{L}=\left(L^{1}, . . L^{n}\right)$ and a density hypothesis between the conditional law of $\boldsymbol{L}$ and the law of $\boldsymbol{L}$. We investigate the link between the global approach and the successive approach.

The application in finance generalizes the default model in Hillairet and Jiao [14] to a dynamic setting. The default time is supposed to be the first time where the firm value reaches a random threshold chosen by the manager of the firm and adjusted dynamically. In literature, another "dynamic" enlargement of filtrations have been introduced by Corcuera et al. [7] where the private information is affected by an independent noise process vanishing as the revelation time approaches. Kchia, Larsson and Protter [23, 22] have studied a progressive filtration expansions with a càdlàg processes. To compare the survival probability for different informations, we introduce the standard 
information available by an agent in credit risk given by the progressive enlargement which has been studied among others, by Jeulin and Yor [20], Mansuy and Yor [24] and Bielecki, Jeanblanc and Rutkowski (e.g [5, 4]) for its application in finance and credit risk. Using our successive enlargement framework, we obtain explicit formulations for the survival probability of the insider and compare the results with those of standard investors by numerical illustrations. Finally we note a strain of related literature dealing with initial enlargement and the information drift such as applying Malliavin's calculus by Imkeller [15, 16, or using forward anticipative calculus by Biagini and Øksendal [3, which provide other perspectives to study the insider information.

The paper is organized as follows. We present the model framework in Section 2. Section 3 introduces the successive density hypothesis and proposes two constructions of auxiliary probability measures to compute conditional expectations. Then, Section 4 considers several particular cases of the successive density framework and makes comparisons. Finally Section 5 applies this insider information framework to a default model and performs some numerical illustrations.

\section{Model framework}

Let $(\Omega, \mathcal{A}, \mathbb{P})$ be a probability space equipped with a reference filtration $\mathbb{F}=\left(\mathcal{F}_{t}\right)_{0 \leq t \leq T}$ which satisfies the usual conditions and represents the common information flow on financial market, where $T$ is a finite time horizon. The insider has knowledge of extra information which are revealed dynamically with time. Let $\left\{t_{i}, i=1, \cdots, n\right\}$ be a family of discrete times ${ }^{1}$ such that $0=t_{1}<\cdots<t_{n}<T$. By convention we set $t_{n+1}=T$. The insider's information is described by a family of random variables $\left\{L^{i}, i=1, \cdots, n\right\}$ where $L^{i}$ is $\mathcal{A}$-measurable and takes values in a Polish space $E$ whose Borel $\sigma$-algebra is denoted by $\mathcal{E}$. The insider gets the information on $L^{i}$ at time $t_{i}$, so the total information flow of the insider is described by the filtration $\mathbb{G}^{I}=\left(\mathcal{G}_{t}^{I}\right)_{t \geq 0}$ where

$$
\mathcal{G}_{t}^{I}:=\mathcal{F}_{t} \vee \sigma\left(L^{1}\right) \vee \cdots \vee \sigma\left(L^{i}\right), \quad t \in\left[t_{i}, t_{i+1}\right)
$$

We can interpret this information flow in two different but equivalent ways by using the theory of enlargement of filtrations. On the one hand, for any $t \in[0, T]$, we can define an extra information process as

$$
L_{t}=\sum_{i=1}^{n} L^{i} 1_{\left[t_{i}, t_{i+1}\right)}(t)
$$

then we have $\mathcal{G}_{t}^{I}=\mathcal{F}_{t} \vee \sigma\left(L_{s}, s \leq t\right)$. The filtration $\mathbb{G}^{I}$ is the progressive enlargement of the filtration $\mathbb{F}$ by the information process $L$. On the other hand, let us define a family of filtrations $\mathbb{G}^{i}=\left(\mathcal{G}_{t}^{i}\right)_{t \geq 0}$, for all $i=1, \cdots, n$, where

$$
\mathcal{G}_{t}^{i}:=\mathcal{F}_{t} \vee \sigma\left(L^{1}\right) \vee \cdots \vee \sigma\left(L^{i}\right), \quad t \in[0, T] .
$$

\footnotetext{
${ }^{1}$ The case of random times $t_{i}$ will be done in a future work.
} 
By definition, we have $\mathcal{G}_{t}^{I}=\mathcal{G}_{t}^{i}$ for $t \in\left[t_{i}, t_{i+1}\right)$ and $\mathcal{G}_{t}^{i}=\mathcal{G}_{t}^{i-1} \vee \sigma\left(L^{i}\right)$, where we set by convention $\mathcal{G}_{t}^{0}=\mathcal{F}_{t}$. Each filtration $\mathbb{G}^{i}$ is the initial enlargement of the filtration $\mathbb{G}^{i-1}$ by the random variable $L^{i}$. We thus obtain an increasing family of successive initial enlargement of filtrations.

We denote by $\boldsymbol{L}$ the $n$ dimensional random vector $\left(L^{1}, \cdots, L^{n}\right)$. For any $i=1, \cdots, n$, let $\boldsymbol{L}^{(i)}:=\left(L^{1}, \cdots, L^{i}\right)$. Similarly, we use the expression $\boldsymbol{x}$ to denote a vector $\left(x^{1}, \cdots, x^{n}\right)$ in $E^{n}$, and let $\boldsymbol{x}^{(i)}:=\left(x^{1}, \cdots, x^{i}\right)$. For any $t \in[0, T]$, the $\sigma$-algebra $\mathcal{G}_{t}^{i}$ is generated by $\mathcal{F}_{t}$ and $\sigma\left(\boldsymbol{L}^{(i)}\right)$. Therefore any $\mathbb{G}^{i}$-adapted process can be written in the form $\left(Y_{t}\left(\boldsymbol{L}^{(i)}\right), 0 \leq t \leq T\right)$ where $Y_{t}(\cdot)$ is $\mathcal{F}_{t} \otimes \mathcal{E}^{\otimes i}$-measurable (c.f. Jeulin [19, Lemma 3.13]).

In the classical framework of initial information modeling, the insider obtains the extra information at the initial time $t=0$ and keeps it until the final time $T$. This corresponds in our setting to the case where $n=1$ and $\mathcal{G}_{t}^{I}=\mathcal{G}_{t}^{1}$ for all $t \in[0, T]$.

In the enlargement of filtration theory, the conditional laws of $L^{i}$ with respect to different filtrations play an important role. For a random variable $X$ taking values in the Polish space $E$ and a sub- $\sigma$-algebra $\mathcal{B}$ of $\mathcal{A}$, we denote by $\mathbb{P}(X \in \cdot \mid \mathcal{B})$ a regular version of the conditional probability law of $X$ with respect to $\mathcal{B}$. By definition, it is a map from $\Omega \times \mathcal{B}$ to $[0,1]$ such that

(1) for almost $\omega \in \Omega, \mathbb{P}(X \in \cdot \mid \mathcal{B})(\omega)$ is a probability measure on $(E, \mathcal{E})$;

(2) for any Borel set $S$ in $E$, the function $\mathbb{P}(X \in S \mid \mathcal{B})$ on $\Omega$ is $\mathcal{B}$-measurable, and is $\mathbb{P}$-a.s. equal to the $\mathcal{B}$-conditional expectation $\mathbb{E}^{\mathbb{P}}\left[1_{S}(X) \mid \mathcal{B}\right]$.

\section{Successive density hypothesis}

In order to study the dynamic properties of the filtration $\mathbb{G}^{I}$, we introduce the following successive density hypothesis, which asserts that the terminal conditional law of $L^{i}$ is equivalent to its $\mathcal{G}_{t_{i}}^{i-1}$ conditional law. This hypothesis is slightly different from Jacod's hypothesis in [18] for the initial enlargement of filtration. The key point is that we take into account the insider's information in a progressive manner at each time step.

Assumption 1 For any $i \in\{1, \cdots, n\}$, the $\mathcal{G}_{T}^{i-1}$-conditional law of $L^{i}$ is equivalent to its $\mathcal{G}_{t_{i}}^{i-1}$ conditional law under the probability $\mathbb{P}$, namely there exists a positive $\mathcal{G}_{T}^{i-1} \otimes \mathcal{E}$-measurable function $\alpha_{T}^{i \mid i-1}\left(\boldsymbol{L}^{(i-1)}, \cdot\right)$ such that

$$
\mathbb{P}\left(L^{i} \in d x \mid \mathcal{G}_{T}^{i-1}\right)=\alpha_{T}^{i \mid i-1}\left(\boldsymbol{L}^{(i-1)}, x\right) \mathbb{P}\left(L^{i} \in d x \mid \mathcal{G}_{t_{i}}^{i-1}\right) \text { a.s.. }
$$

Remark 3.1 1) In the above assumption, we actually consider the density $\alpha_{T}^{i \mid i-1}\left(\boldsymbol{L}^{(i-1)}, \cdot\right)$ as an $\left(\mathcal{F}_{T} \otimes \mathcal{E}^{\otimes i-1}\right) \otimes \mathcal{E}$-measurable function $\alpha_{T}^{i \mid i-1}(\cdot, \cdot)$ evaluated at $\boldsymbol{L}^{(i-1)}$. Note that such representation needs not to be unique. More precisely, there may exist another $\left(\mathcal{F}_{T} \otimes \mathcal{E}^{\otimes i-1}\right) \otimes \mathcal{E}$ measurable function $\widetilde{\alpha}_{T}^{i \mid i-1}(\cdot, \cdot)$ such that $\widetilde{\alpha}_{T}^{i \mid i-1}\left(\boldsymbol{x}^{(i-1)}, x\right)$ is not identically equal to $\alpha_{T}^{i \mid i-1}\left(\boldsymbol{x}^{(i-1)}, x\right)$ for $\left(\boldsymbol{x}^{(i-1)}, x\right) \in E^{i}$ but $\widetilde{\alpha}_{T}^{i \mid i-1}\left(\boldsymbol{L}^{(i-1)}, x\right)=\alpha_{T}^{i \mid i-1}\left(\boldsymbol{L}^{(i-1)}, x\right)$. We refer the readers to [25] for 
a general discussion on the stochastic process depending on a parameter, see also [9, §3.2] for more details on the link with such conditional density processes.

2) In Jacod's hypothesis (see [18]), it is assumed that the $\mathcal{G}_{t}^{i-1}$-conditional law of $L^{i}$ is equivalent to its probability law where $t \in \mathbb{R}_{+}$. Rather than assuming Assumption 1 for $\mathbb{P}\left(L^{i} \in d x \mid \mathcal{G}_{t}^{i-1}\right)$, in our setting, we consider a finite terminal time horizon $T$. The main difference here with Jacod's hypothesis is that the conditional law $\mathbb{P}\left(L^{i} \in d x \mid \mathcal{G}_{t_{i}}^{i-1}\right)$ itself is a random measure instead of a deterministic probability law. Therefore, it is difficult to apply Jacod's method [18, Lemma 1.8] to prove the existence of a martingale version of the density process. Our choice of working with the terminal time $T$ allows to overcome this difficulty. In fact, Assumption 1 implies that, for any $t \in\left[t_{i}, T\right]$, the $\mathcal{G}_{t}^{i-1}$-conditional law of $L^{i}$ under $\mathbb{P}$ is equivalent to the $\mathcal{G}_{t_{i}}^{i-1}$-conditional law of $L^{i}$. Moreover, the $\mathcal{G}_{t}^{i-1} \otimes \mathcal{E}$-measurable function $\mathbb{E}^{\mathbb{P}}\left[\alpha_{T}^{i \mid i-1}\left(\boldsymbol{L}^{(i-1)}, x\right) \mid \mathcal{G}_{t}^{i-1}\right]$ gives the density of $\mathbb{P}\left(L^{i} \in d x \mid \mathcal{G}_{t}^{i-1}\right)$ with respect to $\mathbb{P}\left(L^{i} \in d x \mid \mathcal{G}_{t_{i}}^{i-1}\right)$, which we denote as $\alpha_{t}^{i \mid i-1}\left(\boldsymbol{L}^{(i-1)}, \cdot\right)$. We refer the reader to Corollary 3.5 for details.

3) Under Assumption 1, similar as in Amendinger [1, Proposition 3.3], the filtration $\mathbb{G}^{i}$ is rightcontinuous on $\left[t_{i}, T\right]$, and also is $\mathbb{G}^{I}$ on $[0, T]$, so all conditional expectations are taken with respect to right-continuous filtrations.

\subsection{One step enlargement of filtration}

The filtration $\mathbb{G}^{I}$ can be considered as a step-by-step enlargement of $\mathbb{F}$. Also the successive density hypothesis has an inductive nature. In this subsection, we focus on one step of the enlargement and develop tools which will be useful in the inductive study of $\mathbb{G}^{I}$.

Let $(\Omega, \mathcal{A}, \mathbb{P})$ be a probability space and $\mathbb{H}=\left(\mathcal{H}_{u}\right)_{u \in[t, T]}$ be a filtration of $\mathcal{A}$, where $t$ is a fixed real number such that $0 \leq t<T$. Let $X$ be an $\mathcal{A}$-measurable random variable which takes value in a Polish space $(E, \mathcal{E})$. We assume that there exists a positive $\mathcal{H}_{T} \otimes \mathcal{E}$-measurable function $q_{T}(\cdot)$ such that

$$
\mathbb{P}\left(X \in d x \mid \mathcal{H}_{T}\right)=q_{T}(x) \mathbb{P}\left(X \in d x \mid \mathcal{H}_{t}\right) .
$$

We denote the conditional distribution $\nu_{t}(d x):=\mathbb{P}\left(X \in d x \mid \mathcal{H}_{t}\right)$.

Example 3.2 We give a simple but illustrative example which satisfies the hypothesis (3.2) but not Jacod's hypothesis. Let $Y_{1}$ and $Y_{2}$ be two independent random variables which both follow the standard normal distribution. Let $X=\max \left(Y_{1}, Y_{2}\right)$. We consider the filtration $\mathbb{H}=\left(\mathcal{H}_{u}\right)_{u \in[t, T]}$ such that $\mathcal{H}_{u}=\sigma\left(Y_{1}\right)$ for all $u \in[t, T]$. It is clear that the $\mathcal{H}_{T}$-conditional law of $X$ has a density w.r.t. the $\mathcal{H}_{t}$-conditional law, which equals to the constant 1 . However it is not true that this conditional law is absolutely continuous w.r.t. the probability law of $X$. In fact, if we denote respectively by $\Phi$ and $\phi$ the probability distribution function and the probability density function of the standard normal distribution, then the probability law of $X$ has the probability density $2 \Phi \phi$. However, the $\sigma\left(Y_{1}\right)$-conditional law of $X$ is $\Phi\left(Y_{1}\right) \delta_{Y_{1}}(d u)+1_{\left[Y_{1},+\infty\right)} \phi(u) d u$, which is not absolutely continuous w.r.t. the Lebesgue measure. This is a typical situation which we can not handle within the classical framework of Jacod's density hypothesis. 
Remark 3.3 The condition 3.2 is invariant under a change of probability measure. Indeed, if $\mathbb{P}^{\prime}$ is an equivalent probability measure with respect to $\mathbb{P}$ with $d \mathbb{P}^{\prime} / d \mathbb{P}=Q_{T}(X)$ on $\mathcal{H}_{T} \vee \sigma(X)$, where $Q_{T}(\cdot)$ is a positive $\mathcal{H}_{T} \otimes \mathcal{E}$-measurable function, then for any non-negative Borel function $f$ on $E$,

$$
\mathbb{E}^{\mathbb{P}^{\prime}}\left[f(X) \mid \mathcal{H}_{T}\right]=\frac{\mathbb{E}^{\mathbb{P}}\left[f(X) Q_{T}(X) \mid \mathcal{H}_{T}\right]}{\mathbb{E}^{\mathbb{P}}\left[Q_{T}(X) \mid \mathcal{H}_{T}\right]}=\frac{\int_{E} f(x) Q_{T}(x) q_{T}(x) \nu_{t}(d x)}{\int_{E} Q_{T}(x) q_{T}(x) \nu_{t}(d x)} .
$$

where $\nu_{t}(d x)=\mathbb{P}\left(X \in d x \mid \mathcal{H}_{t}\right)$. Moreover, let $Q_{t}(\cdot)$ be a $\mathcal{H}_{t} \otimes \mathcal{E}$-measurable function such that $Q_{t}(X)=\mathbb{E}^{\mathbb{P}}\left[Q_{T}(X) \mid \mathcal{H}_{t} \vee \sigma(X)\right]$, then $Q_{t}(X)$ is the Radon-Nikodym density $d \mathbb{P}^{\prime} / d \mathbb{P}$ on $\mathcal{H}_{t} \vee \sigma(X)$, and hence

$$
\mathbb{E}^{\mathbb{P}^{\prime}}\left[f(X) \mid \mathcal{H}_{t}\right]=\frac{\mathbb{E}^{\mathbb{P}}\left[f(X) Q_{t}(X) \mid \mathcal{H}_{t}\right]}{\mathbb{E}^{\mathbb{P}}\left[Q_{t}(X) \mid \mathcal{H}_{t}\right]}=\frac{\int_{E} f(x) Q_{t}(x) \nu_{t}(d x)}{\int_{E} Q_{t}(x) \nu_{t}(d x)}
$$

Therefore $\mathbb{P}^{\prime}\left(X \in \cdot \mid \mathcal{H}_{T}\right)$ is absolutely continuous with respect to $\mathbb{P}^{\prime}\left(X \in \cdot \mid \mathcal{H}_{t}\right)$, and the corresponding density is given by

$$
q_{T}^{\prime}(\cdot)=q_{T}(\cdot) \frac{Q_{T}(\cdot)}{Q_{t}(\cdot)} \frac{\int_{E} Q_{t}(x) \nu_{t}(d x)}{\int_{E} Q_{T}(x) q_{T}(x) \nu_{t}(d x)} .
$$

Note that, if $X$ and $\mathcal{H}_{T}$ are $\mathbb{P}$-conditionally independent given $\mathcal{H}_{t}$, then we can choose $Q_{t}(\cdot)$ to be

$$
Q_{t}(\cdot):=\mathbb{E}^{\mathbb{P}}\left[Q_{T}(\cdot) \mid \mathcal{H}_{t}\right]
$$

Let $\mathbb{G}=\left(\mathcal{G}_{u}\right)_{u \in[t, T]}$ denote the initial enlargement of $\mathbb{H}$ with $X$, i.e., $\mathcal{G}_{u}=\mathcal{H}_{u} \vee \sigma(X)$. By using the conditional density, one can construct a probability measure equivalent to $\mathbb{P}$ under which the random variable $X$ and the filtration $\mathbb{H}$ are conditionally independent given $\mathcal{H}_{t}$.

Proposition 3.4 Under hypothesis (3.2), there exists an equivalent probability measure $\mathbb{Q}$ with respect to $\mathbb{P}$ such that

1) $\mathbb{Q}$ coincides with $\mathbb{P}$ on $\mathbb{H}$,

2) $X$ and $\mathbb{H}$ are conditionally independent under $\mathbb{Q}$ given $\mathcal{H}_{t}$,

3) $X$ has the same conditional law given $\mathcal{H}_{t}$ under $\mathbb{P}$ and $\mathbb{Q}$.

Moreover, the probability measure $\mathbb{Q}$ is unique on $\mathcal{G}_{T}$ and given by $\left.\frac{d \mathbb{Q}}{d \mathbb{P}}\right|_{\mathcal{G}_{T}}=q_{T}(X)^{-1}$.

We emphasize that, although the result has a form similar as in Föllmer and Imkeller [11] and Grorud and Pontier [12, under our hypothesis it is in general not possible to expect the independence between $X$ and the filtration $\mathbb{H}$ under an equivalent probability measure.

Proof: By taking the expectation of a conditional expectation, we have

$$
\mathbb{E}^{\mathbb{P}}\left[q_{T}(X)^{-1}\right]=\mathbb{E}^{\mathbb{P}}\left[\mathbb{E}^{\mathbb{P}}\left[q_{T}(X)^{-1} \mid \mathcal{H}_{T}\right]\right]=\mathbb{E}^{\mathbb{P}}\left[\int_{E} q_{T}(x)^{-1} \nu_{T}(d x)\right] .
$$


The hypothesis $(3.2)$ thus leads to

$$
\mathbb{E}^{\mathbb{P}}\left[q_{T}(X)^{-1}\right]=\mathbb{E}^{\mathbb{P}}\left[\int_{E} q_{T}(x)^{-1} q_{T}(x) \nu_{t}(d x)\right]=1
$$

Let $\mathbb{Q}$ be the probability measure on $(\Omega, \mathcal{A})$ defined by $d \mathbb{Q} / d \mathbb{P}=q_{T}(X)^{-1}$. If $f$ is a non-negative Borel function on $E, Z_{T}$ a non-negative $\mathcal{H}_{T}$-measurable random variable and $Y_{t}$ a non-negative $\mathcal{H}_{t}$-measurable random variable, then a direct computation shows

$$
\begin{aligned}
\mathbb{E}^{\mathbb{Q}}\left[f(X) Z_{T} Y_{t}\right] & =\mathbb{E}^{\mathbb{P}}\left[f(X) q_{T}(X)^{-1} Z_{T} Y_{t}\right]=\mathbb{E}^{\mathbb{P}}\left[Z_{T} Y_{t} \int_{E} f(x) q_{T}(x)^{-1} \nu_{T}(d x)\right] \\
& =\mathbb{E}^{\mathbb{P}}\left[Z_{T} Y_{t} \int_{E} f(x) \nu_{t}(d x)\right]=\mathbb{E}^{\mathbb{P}}\left[\mathbb{E}^{\mathbb{P}}\left[Z_{T} \mid \mathcal{H}_{t}\right] Y_{t} \mathbb{E}^{\mathbb{P}}\left[f(X) \mid \mathcal{H}_{t}\right]\right] .
\end{aligned}
$$

If we take $Z_{T}$ to be the constant 1 , we obtain that the conditional law of $X$ under $\mathbb{P}$ and $\mathbb{Q}$ given $\mathcal{H}_{t}$ coincide. If we take $f$ and $Y_{t}$ to be the constant function 1 , we obtain that $\mathbb{P}$ and $\mathbb{Q}$ coincide on $\mathcal{H}_{T}$. Therefore the relation (3.4) implies that

$$
\mathbb{E}^{\mathbb{Q}}\left[f(X) Z_{T} \mid \mathcal{H}_{t}\right]=\mathbb{E}^{\mathbb{Q}}\left[f(X) \mid \mathcal{H}_{t}\right] \mathbb{E}^{\mathbb{Q}}\left[Z_{T} \mid \mathcal{H}_{t}\right],
$$

namely $\sigma(X)$ and $\mathbb{H}$ are conditionally independent given $\mathcal{H}_{t}$.

For the unicity of the probability measure $\mathbb{Q}$ on $\mathcal{G}_{T}$, it suffices to observe that, for any positive $\mathcal{G}_{T}$-measurable random variable $Y_{T}(X)$ one has

$$
\mathbb{E}^{\mathbb{Q}}\left[Y_{T}(X)\right]=\mathbb{E}^{\mathbb{Q}}\left[\int_{E} \mathbb{E}^{\mathbb{Q}}\left[Y_{T}(x) \mid \mathcal{H}_{t}\right] \mathbb{Q}\left(X \in d x \mid \mathcal{H}_{t}\right)\right]
$$

by using the conditional independence of $\mathbb{H}$ and $\sigma(X)$ given $\mathcal{H}_{t}$. Since the probability measures $\mathbb{P}$ and $\mathbb{Q}$ coincide on $\mathbb{H}$ and the conditional probability laws of $X$ given $\mathcal{H}_{t}$ with respect to $\mathbb{P}$ and $\mathbb{Q}$ coincide, one obtains

$$
\begin{aligned}
\mathbb{E}^{\mathbb{Q}}\left[Y_{T}(X)\right] & =\mathbb{E}^{\mathbb{P}}\left[\int_{E} \mathbb{E}^{\mathbb{P}}\left[Y_{T}(x) \mid \mathcal{H}_{t}\right] \mathbb{P}\left(X \in d x \mid \mathcal{H}_{t}\right)\right]=\mathbb{E}^{\mathbb{P}}\left[\int_{E} Y_{T}(x) \mathbb{P}\left(X \in d x \mid \mathcal{H}_{t}\right)\right] \\
& =\mathbb{E}^{\mathbb{P}}\left[\int_{E} Y_{T}(x) q_{T}(x)^{-1} \mathbb{P}\left(X \in d x \mid \mathcal{H}_{T}\right)\right]=\mathbb{E}^{\mathbb{P}}\left[Y_{T}(X) q_{T}(X)^{-1}\right] .
\end{aligned}
$$

Therefore the Radon-Nikodym density of $\mathbb{Q}$ with respect to $\mathbb{P}$ on $\mathcal{G}_{T}$ should be $q_{T}(X)^{-1}$.

Corollary 3.5 For any $u \in[t, T]$, the $\mathcal{H}_{u}$-conditional law of $X$ is equivalent to the $\mathcal{H}_{t}$-conditional law of $X$ under the probability $\mathbb{P}$. Moreover, if $q_{u}(\cdot)$ is a positive $\mathcal{H}_{u} \otimes \mathcal{E}$-measurable function on $\Omega \times E$ such that $q_{u}(x)=\mathbb{E}^{\mathbb{P}}\left[q_{T}(x) \mid \mathcal{H}_{u}\right] \mathbb{P}$-a.s., then one has

$$
\mathbb{P}\left(X \in d x \mid \mathcal{H}_{u}\right)=q_{u}(x) \mathbb{P}\left(X \in d x \mid \mathcal{H}_{t}\right) .
$$

In particular, the Radon-Nikodym derivative of the probability measure $\mathbb{Q}$ defined in the previous proposition with respect to $\mathbb{P}$ is given by $q_{u}(X)^{-1}$ on $\mathcal{H}_{u}$ for $u \in[t, T]$. 
Proof: Let $\mathbb{Q}$ be the probability measure on $\mathcal{A}$ defined by $d \mathbb{Q} / d \mathbb{P}=q_{T}(X)^{-1}$. By Proposition 3.4 . for any $u \in[t, T]$, we obtain

$$
\mathbb{Q}\left(X \in \cdot \mid \mathcal{H}_{u}\right)=\mathbb{Q}\left(X \in \cdot \mid \mathcal{H}_{t}\right)=\mathbb{P}\left(X \in \cdot \mid \mathcal{H}_{t}\right) .
$$

Moreover, for any non-negative Borel function $f$ on $E$ one has

$$
\int_{E} f(x) \mathbb{P}\left(X \in d x \mid \mathcal{H}_{u}\right)=\mathbb{E}^{\mathbb{P}}\left[f(X) \mid \mathcal{H}_{u}\right]=\frac{\mathbb{E}^{\mathbb{Q}}\left[f(X) q_{T}(X) \mid \mathcal{H}_{u}\right]}{\mathbb{E}^{\mathbb{Q}}\left[q_{T}(X) \mid \mathcal{H}_{u}\right]}
$$

Note that

$$
\begin{aligned}
& \mathbb{E}^{\mathbb{Q}}\left[f(X) q_{T}(X) \mid \mathcal{H}_{u}\right]=\mathbb{E}^{\mathbb{Q}}\left[\int_{E} f(x) q_{T}(x) \mathbb{Q}\left(X \in d x \mid \mathcal{H}_{T}\right) \mid \mathcal{H}_{u}\right] \\
= & \mathbb{E}^{\mathbb{Q}}\left[\int_{E} f(x) q_{T}(x) \nu_{t}(d x) \mid \mathcal{H}_{u}\right]=\mathbb{E}^{\mathbb{Q}}\left[\int_{E} f(x) \nu_{T}(d x) \mid \mathcal{H}_{u}\right],
\end{aligned}
$$

where the second equality comes from (3.5) and we recall $\nu_{t}(d x)=\mathbb{P}\left(X \in d x \mid \mathcal{H}_{t}\right)$. In addition, we have from $(3.7)$ that

$$
\mathbb{E}^{\mathbb{Q}}\left[f(X) q_{T}(X) \mid \mathcal{H}_{u}\right]=\int_{E} f(x) \mathbb{E}^{\mathbb{Q}}\left[q_{T}(x) \mid \mathcal{H}_{u}\right] \nu_{t}(d x)=\int_{E} f(x) q_{u}(x) \nu_{t}(d x)
$$

since $\mathbb{Q}$ and $\mathbb{P}$ coincide on $\mathbb{H}$. In particular, when $f$ is the constant function $1,(3.7)$ shows that

$$
\mathbb{E}^{\mathbb{Q}}\left[q_{T}(X) \mid \mathcal{H}_{u}\right]=1
$$

Therefore by (3.6) and (3.8), we obtain

$$
\int_{E} f(x) \mathbb{P}\left(X \in d x \mid \mathcal{H}_{u}\right)=\int_{E} f(x) q_{u}(x) \mathbb{P}\left(X \in d x \mid \mathcal{H}_{t}\right),
$$

namely $q_{u}(\cdot)$ is the density of $\nu_{u}(d x)$ with respect to $\nu_{t}(d x)$.

\subsection{Change of probability measures}

We now come back to the successive enlargements under Assumption 1. In this subsection and the next one, we introduce two different ways to construct equivalent probability measures, which will play an important role in further applications.

We recall that for any $x \in E$ and $t \in\left[t_{i}, T\right], \alpha_{t}^{i \mid i-1}\left(\boldsymbol{L}^{(i-1)}, \cdot\right)$ is defined as the conditional expectation:

$$
\alpha_{t}^{i \mid i-1}\left(\boldsymbol{L}^{(i-1)}, x\right)=\mathbb{E}^{\mathbb{P}}\left[\alpha_{T}^{i \mid i-1}\left(\boldsymbol{L}^{(i-1)}, x\right) \mid \mathcal{G}_{t}^{i-1}\right] .
$$

By Corollary 3.5, we have

$$
\mathbb{P}\left(L^{i} \in d x \mid \mathcal{G}_{t}^{i-1}\right)=\alpha_{t}^{i \mid i-1}\left(\boldsymbol{L}^{(i-1)}, x\right) \mathbb{P}\left(L^{i} \in d x \mid \mathcal{G}_{t_{i}}^{i-1}\right) .
$$

We now introduce a family of probability measures equivalent to $\mathbb{P}$ by using Proposition 3.4 in a recursive manner. 
Definition 3.6 Let $\mathbb{P}^{0}:=\mathbb{P}$, and for any $i \in\{1, \cdots, n\}$, let $\mathbb{P}^{i}$ be the probability measure on $(\Omega, \mathcal{A})$ such that

$$
\frac{d \mathbb{P}^{i}}{d \mathbb{P}^{i-1}}=\frac{1}{\alpha_{T}^{i \mid i-1}\left(\boldsymbol{L}^{(i)}\right)} .
$$

Obviously $\alpha_{T}^{i \mid i-1}\left(\boldsymbol{L}^{(i)}\right)=\alpha_{T}^{i \mid i-1}\left(\boldsymbol{L}^{(i-1)}, L^{i}\right)$. For any $\boldsymbol{x}^{(i)} \in E^{i}$, let

$$
\psi_{t}^{i}\left(\boldsymbol{x}^{(i)}\right):=\prod_{k=1}^{i} \frac{1}{\alpha_{t}^{k \mid k-1}\left(\boldsymbol{x}^{(k)}\right)}, \quad t \in\left[t_{i}, T\right]
$$

We show in Proposition 3.7 below that the probability measures $\left(\mathbb{P}^{i}\right)_{i=1}^{n}$ are well defined and the Radon-Nikodym density of $\mathbb{P}^{i}$ with respect to $\mathbb{P}$ is equal to $\psi_{t}^{i}\left(\boldsymbol{L}^{(i)}\right)$ on $\mathcal{G}_{t}^{i}$.

Proposition 3.7 The probability measures $\left(\mathbb{P}^{i}\right)_{i=1}^{n}$ are well defined and equivalent to $\mathbb{P}$. For any $i \in\{1, \ldots, n\}$,

1) the probability measures $\mathbb{P}^{i}$ and $\mathbb{P}^{i-1}$ coincide on $\mathcal{G}_{T}^{i-1}$, in particular, all probability measures $\left(\mathbb{P}^{i}\right)_{i=1}^{n}$ coincide with $\mathbb{P}$ on $\mathcal{F}_{T}$,

2) $\boldsymbol{L}^{(i)}$ and $\mathcal{F}_{T}$ are conditionally independent given $\mathcal{F}_{t_{i}}$ under $\mathbb{P}^{i}$,

3) for any $t \in\left[t_{i}, T\right]$, the Radon-Nikodym density of $\mathbb{P}^{i}$ w.r.t. $\mathbb{P}^{i-1}$ is given by $\left[\alpha_{t}^{i \mid i-1}\left(\boldsymbol{L}^{(i)}\right)\right]^{-1}$ on $\mathcal{G}_{t}^{i}$ and hence the Radon-Nikodym density of $\mathbb{P}^{i}$ w.r.t. $\mathbb{P}$ is equal to $\psi_{t}^{i}\left(\boldsymbol{L}^{(i)}\right)$ on $\mathcal{G}_{t}^{i}$.

Proof: We prove the proposition by induction on $i$. The case when $i=1$ is true by Proposition 3.4. Suppose that the equivalent probability measures $\mathbb{P}^{1}, \cdots, \mathbb{P}^{i-1}$ are well defined and verify the properties asserted by the proposition. Moreover, Assumption 1 holds for the probability measure $\mathbb{P}^{i-1}$ by Remark 3.3. More precisely, the conditional law $\mathbb{P}^{i-1}\left(L^{i} \in \cdot \mid \mathcal{G}_{T}^{i-1}\right)$ is absolutely continuous w.r.t. $\mathbb{P}^{i-1}\left(L^{i} \in \cdot \mid \mathcal{G}_{t_{i}}^{i-1}\right)$, and the corresponding density is

$$
\alpha_{T}^{i \mid i-1}\left(\boldsymbol{L}^{(i-1)}, \cdot\right) \frac{\psi_{T}^{i-1}\left(\boldsymbol{L}^{(i-1)}\right)}{\mathbb{E}^{\mathbb{P}}\left[\psi_{T}^{i-1}\left(\boldsymbol{L}^{(i-1)}\right) \mid \mathcal{G}_{t_{i}}^{i-1}\right]} \frac{\int_{E} \mathbb{E}^{\mathbb{P}}\left[\psi_{T}^{i-1}\left(\boldsymbol{L}^{(i-1)}\right) \mid \mathcal{G}_{t_{i}}^{i-1}\right] \mathbb{P}\left(L^{i} \in d x \mid \mathcal{G}_{t_{i}}^{i-1}\right)}{\int_{E} \alpha_{T}^{i \mid i-1}\left(\boldsymbol{L}^{(i-1)}, x\right) \psi_{T}^{i-1}\left(\boldsymbol{L}^{(i-1)}\right) \mathbb{P}\left(L^{i} \in d x \mid \mathcal{G}_{t_{i}}^{i-1}\right)},
$$

which is equal to $\alpha_{T}^{i \mid i-1}\left(\boldsymbol{L}^{(i-1)}, \cdot\right)$, since

$$
\psi_{T}^{i-1}\left(\boldsymbol{L}^{(i-1)}\right)=\int_{E} \alpha_{T}^{i \mid i-1}\left(\boldsymbol{L}^{(i-1)}, x\right) \psi_{T}^{i-1}\left(\boldsymbol{L}^{(i-1)}\right) \mathbb{P}\left(L^{i} \in d x \mid \mathcal{G}_{t_{i}}^{i-1}\right)
$$

and

$$
\mathbb{E}^{\mathbb{P}}\left[\psi_{T}^{i-1}\left(\boldsymbol{L}^{(i-1)}\right) \mid \mathcal{G}_{t_{i}}^{i-1}\right]=\int_{E} \mathbb{E}^{\mathbb{P}}\left[\psi_{T}^{i-1}\left(\boldsymbol{L}^{(i-1)}\right) \mid \mathcal{G}_{t_{i}}^{i-1}\right] \mathbb{P}\left(L^{i} \in d x \mid \mathcal{G}_{t_{i}}^{i-1}\right) .
$$

We now show that 3.10 effectively defines a probability measure $\mathbb{P}^{i}$. One has

$$
\mathbb{E}^{\mathbb{P}^{i-1}}\left[\alpha_{T}^{i \mid i-1}\left(\boldsymbol{L}^{(i)}\right)^{-1} \mid \mathcal{G}_{t_{i}}^{i-1}\right]=\frac{\mathbb{E}^{\mathbb{P}}\left[\alpha_{T}^{i \mid i-1}\left(\boldsymbol{L}^{(i)}\right)^{-1} \psi_{T}^{i-1}\left(\boldsymbol{L}^{(i-1)}\right) \mid \mathcal{G}_{t_{i}}^{i-1}\right]}{\mathbb{E}^{\mathbb{P}}\left[\psi_{T}^{i-1}\left(\boldsymbol{L}^{(i-1)}\right) \mid \mathcal{G}_{t_{i}}^{i-1}\right]} .
$$


The assumption (3.1) applied to $L^{i}$ and $\mathbb{G}^{i-1}$ leads to

$$
\begin{aligned}
& \mathbb{E}^{\mathbb{P}}\left[\alpha_{T}^{i \mid i-1}\left(\boldsymbol{L}^{(i)}\right)^{-1} \psi_{T}^{i-1}\left(\boldsymbol{L}^{(i-1)}\right) \mid \mathcal{G}_{t_{i}}^{i-1}\right] \\
= & \mathbb{E}^{\mathbb{P}}\left[\psi_{T}^{i-1}\left(\boldsymbol{L}^{(i-1)}\right) \int_{E} \alpha_{T}^{i \mid i-1}\left(\boldsymbol{L}^{(i-1)}, x\right)^{-1} \alpha_{T}^{i \mid i-1}\left(\boldsymbol{L}^{(i-1)}, x\right) \mathbb{P}\left(L^{i} \in d x \mid \mathcal{G}_{t_{i}}^{i-1}\right) \mid \mathcal{G}_{t_{i}}^{i-1}\right] \\
= & \mathbb{E}^{\mathbb{P}}\left[\psi_{T}^{i-1}\left(\boldsymbol{L}^{(i-1)}\right) \mid \mathcal{G}_{t_{i}}^{i-1}\right] .
\end{aligned}
$$

Therefore $\mathbb{E}^{\mathbb{P}^{i-1}}\left[\alpha_{T}^{i \mid i-1}\left(\boldsymbol{L}^{(i)}\right)^{-1} \mid \mathcal{G}_{t_{i}}^{i-1}\right]=1$ and hence $\mathbb{P}^{i}$ is a well defined probability measure.

By Proposition 3.4, $\mathbb{P}^{i}$ and $\mathbb{P}^{i-1}$ coincide on $\mathbb{G}^{i-1}$. In particular, $\mathbb{P}^{i}$ and $\mathbb{P}$ are the same on $\mathcal{F}_{T}$, which implies the first assertion. By the induction hypothesis, $\boldsymbol{L}^{(i-1)}$ and $\mathcal{F}_{T}$ are conditionally independent given $\mathcal{F}_{t_{i-1}}$ under the probablity measure $\mathbb{P}^{i-1}$, which implies, since $\mathcal{F}_{t_{i-1}} \subseteq \mathcal{F}_{t_{i}}$, that $\boldsymbol{L}^{(i-1)}$ and $\mathcal{F}_{T}$ are conditionnally independent given $\mathcal{F}_{t_{i}}$ under $\mathbb{P}^{i-1}$, and also under $\mathbb{P}^{i}$ by 1$)$. It then suffices to verify that $L^{i}$ and $\mathcal{F}_{T}$ are conditionally independent given $\mathcal{F}_{t_{i}}$ under $\mathbb{P}^{i}$ to prove the second assertion. Note that Proposition 3.4 also shows that $L^{i}$ and $\mathcal{G}_{T}^{i-1}$ are conditionally independent given $\mathcal{G}_{t_{i}}^{i-1}$ under the probability $\mathbb{P}^{\imath}$. Let $f$ be a non-negative Borel function on $E$ and $X$ is a non-negative $\mathcal{F}_{T}$-mesurable random variable. By the conditional independence of $L^{i}$ and $\mathcal{F}_{T}$ given $\mathcal{G}_{t_{i}}^{i-1}$ under $\mathbb{P}^{i}$, one obtains

$$
\mathbb{E}^{\mathbb{P}^{i}}\left[f\left(L^{i}\right) X \mid \mathcal{F}_{t_{i}}\right]=\mathbb{E}^{\mathbb{P}^{i}}\left[\mathbb{E}^{\mathbb{P}^{i}}\left[f\left(L^{i}\right) \mid \mathcal{G}_{t_{i}}^{i-1}\right] \cdot \mathbb{E}^{\mathbb{P}^{i}}\left[X \mid \mathcal{G}_{t_{i}}^{i-1}\right] \mid \mathcal{F}_{t_{i}}\right]
$$

Moreover, since $X$ and $\boldsymbol{L}^{(i-1)}$ are conditionally independent given $\mathcal{F}_{t_{i}}$ under $\mathbb{P}^{i}$, one has $\mathbb{E}^{\mathbb{P}^{i}}\left[X \mid \mathcal{G}_{t_{i}}^{i-1}\right]=$ $\mathbb{E}^{\mathbb{P}^{i}}\left[X \mid \mathcal{F}_{t_{i}}\right]$ (cf. Dellacherie-Meyer [8, theorem 45]). Therefore one obtains

$$
\mathbb{E}^{\mathbb{P}^{i}}\left[f\left(L^{i}\right) X \mid \mathcal{F}_{t_{i}}\right]=\mathbb{E}^{\mathbb{P}^{i}}\left[f\left(L^{i}\right) \mid \mathcal{F}_{t_{i}}\right] \cdot \mathbb{E}^{\mathbb{P}^{i}}\left[X \mid \mathcal{F}_{t_{i}}\right] .
$$

Finally, the last assertion of the proposition follows from 1) and Corollary 3.5. The proposition is thus proved.

Remark 3.8 This construction of successive changes of probability measures is natural and only use the knowledge of $\boldsymbol{L}^{(i)}$ to construct $\mathbb{P}^{i}$. However, under the probability measure $\mathbb{P}^{i}$, the law of $L^{k}, k \in\{i+1, \cdots, n\}$ is not identical to the law of $L^{k}$ under $\mathbb{P}^{i-1}$. We will show in the next subsection that $\mathbb{P}^{n}$ preserves the $\mathbb{P}$-conditional probability law of $L^{k}$ given $\mathcal{G}_{t_{k}}^{k-1}$.

Proposition 3.9 Let $t, u \in\left[t_{i}, T\right], t \leq u$ and $X_{u}\left(\boldsymbol{L}^{(i)}\right)$ be a non-negative $\mathcal{G}_{u}^{i}$-measurable random variable. One has

$$
\mathbb{E}^{\mathbb{P}}\left[X_{u}\left(\boldsymbol{L}^{(i)}\right) \mid \mathcal{G}_{t}^{i}\right]=\left.\frac{\mathbb{E}^{\mathbb{P}}\left[X_{u}\left(\boldsymbol{x}^{(i)}\right) \psi_{u}^{i}\left(\boldsymbol{x}^{(i)}\right)^{-1} \mid \mathcal{F}_{t}\right]}{\psi_{t}^{i}\left(\boldsymbol{x}^{(i)}\right)^{-1}}\right|_{\boldsymbol{x}^{(i)}=\boldsymbol{L}^{(i)}}
$$

Proof: We use the change of the probability measure to $\mathbb{P}^{i}$ and obtain

$$
\mathbb{E}^{\mathbb{P}}\left[X_{u}\left(\boldsymbol{L}^{(i)}\right) \mid \mathcal{G}_{t}^{i}\right]=\frac{\mathbb{E}^{\mathbb{P}^{i}}\left[X_{u}\left(\boldsymbol{L}^{(i)}\right) \psi_{u}^{i}\left(\boldsymbol{L}^{(i)}\right)^{-1} \mid \mathcal{G}_{t}^{i}\right]}{\psi_{t}^{i}\left(\boldsymbol{L}^{(i)}\right)^{-1}}
$$


By Proposition 3.7, $\boldsymbol{L}^{(i)}$ and $\mathcal{F}_{T}$ are conditionally independent given $\mathcal{F}_{t}$ under the probability $\mathbb{P}^{i}$. Therefore

$$
\mathbb{E}^{\mathbb{P}}\left[X_{u}\left(\boldsymbol{L}^{(i)}\right) \mid \mathcal{G}_{t}^{i}\right]=\left.\frac{\mathbb{E}^{\mathbb{P}^{i}}\left[X_{u}\left(\boldsymbol{x}^{(i)}\right) \psi_{u}^{i}\left(\boldsymbol{x}^{(i)}\right)^{-1} \mid \mathcal{F}_{t}\right]}{\psi_{t}^{i}\left(\boldsymbol{x}^{(i)}\right)^{-1}}\right|_{\boldsymbol{x}^{(i)}=\boldsymbol{L}^{(i)}}
$$

Since $\mathbb{P}^{i}$ and $\mathbb{P}$ coincide on $\mathcal{F}_{T}$, we obtain the desired result.

\subsection{Backward construction of probability measures}

In order to have a family of probability measures under which the conditional law of each $L^{i}$ remains unchanged, we propose the following construction, using a backward change of probability measures. This method is also crucial in the evaluation of financial claims which we will discuss later on.

Definition 3.10 Let $\mathbb{Q}^{n+1}=\mathbb{P}$, and for $i \in\{1, \ldots, n\}$, let $\mathbb{Q}^{i}$ be a probability measure on $(\Omega, \mathcal{A})$ such that

$$
\frac{d \mathbb{Q}^{i}}{d \mathbb{Q}^{i+1}}:=\frac{1}{\alpha_{T}^{i \mid i-1}\left(\boldsymbol{L}^{(i)}\right)} .
$$

Let

$$
\varphi_{T}^{i}(\boldsymbol{x})=\prod_{k=i}^{n} \frac{1}{\alpha_{T}^{k \mid k-1}\left(\boldsymbol{x}^{(k)}\right)} .
$$

Then the Radon-Nikodym derivative of $\mathbb{Q}^{i}$ with respect to $\mathbb{P}$ is given by

$$
\frac{d \mathbb{Q}^{i}}{d \mathbb{P}}=\varphi_{T}^{i}(\boldsymbol{L})
$$

Note that $\varphi_{T}^{i}(\boldsymbol{L})$ is a $\mathcal{G}_{T}^{n}$-measurable random variable.

Proposition 3.11 The equivalent probability measures $\left(\mathbb{Q}^{i}\right)_{i=1}^{n}$ are well defined and verify the following properties for any $i \in\{1, \cdots, n\}$

1) $\mathbb{Q}^{i}$ coincides with $\mathbb{P}$ on $\mathcal{G}_{T}^{i-1}$,

2) for any $k \in\{i, \cdots, n\}, L^{k}$ and $\mathcal{G}_{T}^{k-1}$ are conditionally independent given $\mathcal{G}_{t_{k}}^{k-1}$ under $\mathbb{Q}^{i}$,

3) for any $k \in\{1, \cdots, n\}, L^{k}$ has the same conditional law given $\mathcal{G}_{t_{k}}^{k-1}$ under all $\left(\mathbb{Q}^{i}\right)_{i=1}^{n}$ and $\mathbb{P}$.

ProOF: We prove the proposition by a reverse induction on $i$. The assertion is clearly true when $i=n+1$. Assume that the probability measures $\mathbb{Q}^{i+1}, \cdots, \mathbb{Q}^{n+1}$ have been constructed and verify the assertions in the proposition. Since $\mathbb{Q}^{i+1}$ is identical to $\mathbb{P}$ on $\mathcal{G}_{T}^{i}$, one has

$$
\mathbb{Q}^{i+1}\left(L^{i} \in d x \mid \mathcal{G}_{T}^{i-1}\right)=\alpha_{T}^{i \mid i-1}\left(\boldsymbol{L}^{(i-1)}, x\right) \mathbb{Q}^{i+1}\left(L^{i} \in d x \mid \mathcal{G}_{t_{i}}^{i-1}\right) .
$$


In particular, one has

$$
\mathbb{E}^{\mathbb{Q}^{i+1}}\left[\alpha_{T}^{i \mid i-1}\left(\boldsymbol{L}^{(i)}\right) \mid \mathcal{G}_{T}^{i-1}\right]=1 .
$$

Therefore, the probability measure $\mathbb{Q}^{i}$ equivalent to $\mathbb{Q}^{i+1}$ given by $(3.12)$ is well defined.

By (3.14) and Proposition 3.4 the probability measure $\mathbb{Q}^{i}$ coincides with $\mathbb{Q}^{i+1}$, and therefore with $\mathbb{P}$, on $\mathcal{G}_{T}^{i-1}$. So the assertion (1) is proved, and hence for any $k \in\{1, \cdots, i-1\}$, $L^{k}$ has the same conditional law given $\mathcal{G}_{t_{k}}^{k-1}$ under $\mathbb{Q}^{i}$ and $\mathbb{P}$. Moreover, $L^{i}$ is conditionally independent of $\mathcal{G}_{T}^{i-1}$ given $\mathcal{G}_{t_{i}}^{i-1}$ under $\mathbb{Q}^{i}$, and $L^{i}$ has the same conditional probability law given $\mathcal{G}_{t_{i}}^{i-1}$ under $\mathbb{Q}^{i}$ and $\mathbb{Q}^{i+1}$ (and hence under $\mathbb{P}$ also). Finally, for $k \in\{i+1, \cdots, n\}$, let $h$ be a non-negative Borel function on $E$ and $Y$ be a non-negative $\mathcal{G}_{T}^{k-1}$-measurable random variable, then

$$
\begin{aligned}
\mathbb{E}^{\mathbb{Q}^{i}}\left[h\left(L^{k}\right) Y \mid \mathcal{G}_{t_{k}}^{k-1}\right] & =\frac{\mathbb{E}^{\mathbb{Q}^{i+1}}\left[h\left(L^{k}\right) Y \alpha_{T}^{i \mid i-1}\left(\boldsymbol{L}^{(i)}\right)^{-1} \mid \mathcal{G}_{t_{k}}^{k-1}\right]}{\mathbb{E}^{\mathbb{Q}^{i+1}}\left[\alpha_{T}^{i \mid i-1}\left(\boldsymbol{L}^{(i)}\right)^{-1} \mid \mathcal{G}_{t_{k}}^{k-1}\right]} \\
& =\frac{\mathbb{E}^{\mathbb{Q}^{i+1}}\left[h\left(L^{k}\right) \mid \mathcal{G}_{t_{k}}^{k-1}\right] \mathbb{E}^{\mathbb{Q}^{i+1}}\left[Y \alpha_{T}^{i \mid i-1}\left(\boldsymbol{L}^{(i)}\right)^{-1} \mid \mathcal{G}_{t_{k}}^{k-1}\right]}{\mathbb{E}^{\mathbb{Q}^{i+1}}\left[\alpha_{T}^{i \mid i-1}\left(\boldsymbol{L}^{(i)}\right)^{-1} \mid \mathcal{G}_{t_{k}}^{k-1}\right]}
\end{aligned}
$$

since by the induction hypothesis, $L^{k}$ and $\mathcal{G}_{T}^{k-1}$ are conditionally independent given $\mathcal{G}_{t_{k}}^{k-1}$ under $\mathbb{Q}^{i+1}$. Therefore

$$
\mathbb{E}^{\mathbb{Q}^{i}}\left[h\left(L^{k}\right) Y \mid \mathcal{G}_{t_{k}}^{k-1}\right]=\mathbb{E}^{\mathbb{Q}^{i+1}}\left[h\left(L^{k}\right) \mid \mathcal{G}_{t_{k}}^{k-1}\right] \mathbb{E}^{\mathbb{Q}^{i}}\left[Y \mid \mathcal{G}_{t_{k}}^{k-1}\right] .
$$

If we take $Y=1$, then $\mathcal{G}_{t_{k}}^{k-1}$-conditional law of $L^{k}$ under $\mathbb{Q}^{i}$ coincides with that under $\mathbb{Q}^{i+1}$, which proves the assertion (3). Moreover, this also shows

$$
\mathbb{E}^{\mathbb{Q}^{i}}\left[h\left(L^{k}\right) Y \mid \mathcal{G}_{t_{k}}^{k-1}\right]=\mathbb{E}^{\mathbb{Q}^{i}}\left[h\left(L^{k}\right) \mid \mathcal{G}_{t_{k}}^{k-1}\right] \mathbb{E}^{\mathbb{Q}^{i}}\left[Y \mid \mathcal{G}_{t_{k}}^{k-1}\right],
$$

which gives the assertion (2) and completes the proof.

\subsection{Conditional expectation with successive information}

In this subsection, we are interested in the computation of conditional expectations with the insider's successive information. The $\mathbb{G}^{I}$-conditional expectations may represent the dynamic values of a financial claim viewed by the insider. The idea is to make connections with the $\mathbb{F}$ conditional expectations which is easier to deal with in an explicit manner and the result is given in a decomposed form with a regime change at each time $t_{i}$ when a new information is available. We still suppose Assumption 1 for the information flow. In particular, we assume that the insider has the knowledge on the marginal conditional laws $\mathbb{P}\left(L^{i} \in d x \mid \mathcal{G}_{t_{i}}^{i-1}\right), i \in\{1, \ldots, n\}$. We shall present the evaluation formula in terms of $\mathbb{F}$-conditional expectations.

Let $Y_{T}(\boldsymbol{L})$ be a non-negative $\mathcal{G}_{T}^{I}$-measurable random variable. Our purpose is to determine the conditional expectation of $Y_{T}(\boldsymbol{L})$ given the insider's information $\mathcal{G}_{t}^{I}$ at $t \in[0, T]$. Here we work under the initial probability measure $\mathbb{P}$. Note that the method is valid under an equivalent probability measure since Assumption 1 is invariant under equivalent probability change. By 
definition (2.1) and (2.3), we have

$$
\mathbb{E}^{\mathbb{P}}\left[Y_{T}(\boldsymbol{L}) \mid \mathcal{G}_{t}^{I}\right]=\sum_{i=1}^{n} 1_{\left[t_{i}, t_{i+1}\right)}(t) \mathbb{E}^{\mathbb{P}}\left[Y_{T}(\boldsymbol{L}) \mid \mathcal{G}_{t}^{i}\right]=\sum_{i=1}^{n} 1_{\left[t_{i}, t_{i+1}\right)}(t) \mathbb{E}^{\mathbb{P}}\left[Y_{t_{i+1}}\left(\boldsymbol{L}^{(i)}\right) \mid \mathcal{G}_{t}^{i}\right]
$$

where

$$
Y_{t_{i+1}}\left(\boldsymbol{L}^{(i)}\right):=\mathbb{E}^{\mathbb{P}}\left[Y_{T}(\boldsymbol{L}) \mid \mathcal{G}_{t_{i+1}}^{i}\right] .
$$

It then suffices to determine $Y_{t_{i+1}}\left(\boldsymbol{L}^{(i)}\right)$ under Assumption 1. The result is obtained by using a recursive pricing kernel and we use probability measures constructed in the two previous subsections.

For any $i \in\{1, \cdots, n\}$, let $J_{i}$ be the operator which sends a non-negative or bounded $\mathcal{G}_{T}^{i}$ measurable random variable $X_{T}\left(\boldsymbol{L}^{(i)}\right)$ to the following integral

$$
\int_{E} \mathbb{E}^{\mathbb{P}}\left[X_{T}\left(\boldsymbol{L}^{(i-1)}, x^{i}\right) \mid \mathcal{G}_{t_{i}}^{i-1}\right] \mathbb{P}\left(L^{i} \in d x^{i} \mid \mathcal{G}_{t_{i}}^{i-1}\right)
$$

which is a $\mathcal{G}_{t_{i}}^{i-1}$-measurable random variable. Note that by Proposition 3.9 , we have

$$
\mathbb{E}^{\mathbb{P}}\left[X_{T}\left(\boldsymbol{L}^{(i-1)}, x^{i}\right) \mid \mathcal{G}_{t_{i}}^{i-1}\right]=\left.\frac{\mathbb{E}^{\mathbb{P}}\left[X_{T}\left(\boldsymbol{x}^{(i)}\right) \psi_{T}^{i-1}\left(\boldsymbol{x}^{(i-1)}\right)^{-1} \mid \mathcal{F}_{t_{i}}\right]}{\psi_{t_{i}}^{i-1}\left(\boldsymbol{x}^{(i-1)}\right)^{-1}}\right|_{\boldsymbol{x}^{(i-1)}=\boldsymbol{L}^{(i-1)}} .
$$

In other terms, the operator $J_{i}$ can be expressed in terms of $\mathbb{F}$-conditional expectation and integral w.r.t. the $\mathcal{G}_{t_{i}}^{i-1}$-conditional law of $L^{i}$.

This operator can be better understood by using the probability measure $\mathbb{Q}^{i}$ constructed in $\$ 3.3$. In fact, by Proposition 3.11, one has

$$
\mathbb{P}\left(L^{i} \in d x^{i} \mid \mathcal{G}_{t_{i}}^{i-1}\right)=\mathbb{Q}^{i}\left(L^{i} \in d x^{i} \mid \mathcal{G}_{t_{i}}^{i-1}\right),
$$

and

$$
\mathbb{E}^{\mathbb{P}}\left[X_{T}\left(\boldsymbol{L}^{(i-1)}, x^{i}\right) \mid \mathcal{G}_{t_{i}}^{i-1}\right]=\mathbb{E}^{\mathbb{Q}^{i}}\left[X_{T}\left(\boldsymbol{L}^{(i-1)}, x^{i}\right) \mid \mathcal{G}_{t_{i}}^{i-1}\right]
$$

since $\mathbb{Q}^{i}$ and $\mathbb{P}$ coincide on $\mathcal{G}_{T}^{i-1}$. Therefore we can rewrite (3.17) as

$$
\int_{E} \mathbb{E}^{\mathbb{Q}^{i}}\left[X_{T}\left(\boldsymbol{L}^{(i-1)}, x^{i}\right) \mid \mathcal{G}_{t_{i}}^{i-1}\right] \mathbb{Q}^{i}\left(L^{i} \in d x^{i} \mid \mathcal{G}_{t_{i}}^{i-1}\right),
$$

which implies, since $L^{i}$ and $\mathcal{G}_{T}^{(i-1)}$ are conditionally independent given $\mathcal{G}_{t_{i}}^{i-1}$ under $\mathbb{Q}^{i}$, that

$$
J_{i}\left(X_{T}\left(\boldsymbol{L}^{(i)}\right)\right)=\mathbb{E}^{\mathbb{Q}^{i}}\left[X_{T}\left(\boldsymbol{L}^{(i)}\right) \mid \mathcal{G}_{t_{i}}^{i-1}\right] .
$$

Therefore, $J_{i}$ is actually a conditional expectation operator. In particular, it is an linear operator which verifies the following equality

$$
J_{i}\left(X_{T}\left(\boldsymbol{L}^{(i)}\right) Z_{t_{i}}\left(\boldsymbol{L}^{(i-1)}\right)\right)=Z_{t_{i}}\left(\boldsymbol{L}^{(i-1)}\right) J_{i}\left(X_{T}\left(\boldsymbol{L}^{(i)}\right)\right)
$$

for any $\mathcal{G}_{t_{i}}^{i-1}$-measurable random variable $Z_{t_{i}}\left(\boldsymbol{L}^{(i-1)}\right)$ such that the left-hand side of the above formula is well defined. 
Lemma 3.12 Let $X_{T}(\boldsymbol{L})$ be a bounded or non-negative $\mathcal{G}_{T}^{n}$-measurable random variable. One has

$$
\mathbb{E}^{\mathbb{Q}^{i+1}}\left[X_{T}(\boldsymbol{L}) \mid \mathcal{G}_{t_{i+1}}^{i}\right]=J_{i+1} \circ \cdots \circ J_{n}\left(X_{T}(\boldsymbol{L}) U_{T}^{i+1}(\boldsymbol{L})\right), \quad i \in\{0, \ldots, n\}
$$

where the operator $J_{i+1} \circ \cdots \circ J_{n}$ is considered as the identity operator when $i=n$ and

$$
U_{T}^{i+1}(\boldsymbol{L}):=\prod_{k=i+1}^{n} \frac{\left.\alpha_{t_{k+1}^{k \mid k-1}}^{k \mid \boldsymbol{L}^{(k)}}\right)}{\alpha_{T}^{k \mid k-1}\left(\boldsymbol{L}^{(k)}\right)} .
$$

Proof: We prove the assertion by reverse induction on $i$. The case when $i=n$ follows from (3.19) since $U_{T}^{n}(\boldsymbol{L})=1$. In the following, we assume that the equality (3.21) is verified for $i+1$ and we now prove it is the case for $i$.

By the induction hypothesis and the fact that $U_{T}^{i+1}(\boldsymbol{L})=\frac{\alpha_{t_{i+2}}^{i+1 \mid i}\left(\boldsymbol{L}^{(i+1)}\right)}{\alpha_{T}^{i+1 \mid i}\left(\boldsymbol{L}^{(i+1)}\right)} U_{T}^{i+2}(\boldsymbol{L})$, one has

$$
\begin{aligned}
& J_{i+1} \circ \cdots \circ J_{n}\left(X_{T}(\boldsymbol{L}) U_{T}^{i+1}(\boldsymbol{L})\right)=J_{i+1}\left(\mathbb{E}^{\mathbb{Q}^{i+2}}\left[X_{T}(\boldsymbol{L}) \frac{\alpha_{t_{i+2}}^{i+1 \mid i}\left(\boldsymbol{L}^{(i+1)}\right)}{\alpha_{T}^{i+1 \mid i}\left(\boldsymbol{L}^{(i+1)}\right)} \mid \mathcal{G}_{t_{i+2}}^{i+1}\right]\right) \\
= & J_{i+1}\left(\mathbb{E}^{\mathbb{Q}^{i+1}}\left[X_{T}(\boldsymbol{L}) \mid \mathcal{G}_{t_{i+2}}^{i+1}\right]\right)=\mathbb{E}^{\mathbb{Q}^{i+1}}\left[X_{T}(\boldsymbol{L}) \mid \mathcal{G}_{t_{i+1}}^{i}\right]
\end{aligned}
$$

where the second equality comes from the probability change from $\mathbb{Q}^{i+2}$ to $\mathbb{Q}^{i+1}$, and the last equality follows from $(3.19)$.

Theorem 3.13 Let $Y_{T}(\boldsymbol{L})$ be a bounded or non-negative $\mathcal{G}_{T}^{I}$-measurable random variable. For any $t \in[0, T]$, we have

$$
\mathbb{E}^{\mathbb{P}}\left[Y_{T}(\boldsymbol{L}) \mid \mathcal{G}_{t}^{I}\right]=\left.\sum_{i=1}^{n} 1_{\left[t_{i}, t_{i+1}\right)}(t) \frac{\mathbb{E}^{\mathbb{P}}\left[Y_{t_{i+1}}\left(\boldsymbol{x}^{(i)}\right) \psi_{t_{i+1}}^{i}\left(\boldsymbol{x}^{(i)}\right)^{-1} \mid \mathcal{F}_{t}\right]}{\psi_{t_{i}}^{i}\left(\boldsymbol{x}^{(i)}\right)^{-1}}\right|_{\boldsymbol{x}^{(i)}=\boldsymbol{L}^{(i)}}
$$

where $Y_{t_{i+1}}(\cdot)$ is $\mathcal{F}_{t_{i+1}} \otimes \mathcal{E}^{\otimes i}$-measurable such that $Y_{t_{i+1}}\left(\boldsymbol{L}^{(i)}\right)=\mathbb{E}^{\mathbb{P}}\left[Y_{T}(\boldsymbol{L}) \mid \mathcal{G}_{t_{i+1}}^{i}\right]$. Moreover, the sequence of random variables $\left(Y_{t_{i+1}}\left(\boldsymbol{L}^{(i)}\right)\right)_{i=0}^{n}$ satisfies the following backward recursive relation

$$
Y_{t_{i+1}}\left(\boldsymbol{L}^{(i)}\right)=\frac{J_{i+1}\left(Y_{t_{i+2}}\left(\boldsymbol{L}^{(i+1)}\right) \Phi_{t_{i+2}}\left(\boldsymbol{L}^{(i+1)}\right)\right)}{J_{i+1}\left(\Phi_{t_{i+2}}\left(\boldsymbol{L}^{(i+1)}\right)\right)}, \quad i \in\{0, \cdots, n-1\},
$$

with the terminal term $Y_{t_{n+1}}\left(\boldsymbol{L}^{(n)}\right)=Y_{T}(\boldsymbol{L})$ and the pricing kernel given by

$$
\Phi_{t_{i+2}}\left(\boldsymbol{L}^{(i+1)}\right):=J_{i+2} \circ \cdots \circ J_{n}\left(\alpha_{t_{i+2}}^{i+1 \mid i}\left(\boldsymbol{L}^{(i)}\right) \cdots \alpha_{T}^{n \mid n-1}\left(\boldsymbol{L}^{(n)}\right)\right)
$$

with convention $\Phi_{t_{1}}=1$.

Proof: By (3.15) and Proposition 3.9, we obtain the equality (3.23). We now prove the relation (3.24) by computing the conditional expectation (3.16) under the change of probability measure to $\mathbb{Q}^{2+1}$ as

$$
Y_{t_{i+1}}\left(\boldsymbol{L}^{(i)}\right)=\mathbb{E}^{\mathbb{P}}\left[Y_{t_{i+2}}\left(\boldsymbol{L}^{(i+1)}\right) \mid \mathcal{G}_{t_{i+1}}^{i}\right]=\frac{\mathbb{E}^{\mathbb{Q}^{i+1}}\left[Y_{t_{i+2}}\left(\boldsymbol{L}^{(i+1)}\right) \varphi_{T}^{i+1}(\boldsymbol{L})^{-1} \mid \mathcal{G}_{t_{i+1}}^{i}\right]}{\mathbb{E}^{\mathbb{Q}^{i+1}}\left[\varphi_{T}^{i+1}(\boldsymbol{L})^{-1} \mid \mathcal{G}_{t_{i+1}}^{i}\right]}
$$


where $\varphi_{T}^{i+1}(\boldsymbol{L})$ is the Radon-Nikodym derivative of $\mathbb{Q}^{i+1}$ with respect to $\mathbb{P}$ defined in 3.13$)$. By Lemma 3.12 , one has

$$
\begin{aligned}
\mathbb{E}^{\mathbb{Q}^{i+1}}\left[Y_{t_{i+2}}\left(\boldsymbol{L}^{(i+1)}\right) \varphi_{T}^{i+1}(\boldsymbol{L})^{-1} \mid \mathcal{G}_{t_{i+1}}^{i}\right] & =J_{i+1} \circ \cdots \circ J_{n}\left(Y_{t_{i+2}}\left(\boldsymbol{L}^{(i+1)}\right) \varphi_{T}^{i+1}(\boldsymbol{L})^{-1} U_{T}^{i+1}(\boldsymbol{L})\right) \\
& =J_{i+1}\left(Y_{t_{i+2}}\left(\boldsymbol{L}^{(i+1)}\right) \cdot J_{i+2} \circ \cdots \circ J_{n}\left(\varphi_{T}^{i+1}(\boldsymbol{L})^{-1} U_{T}^{i+1}(\boldsymbol{L})\right)\right.
\end{aligned}
$$

where the second equality comes from $(3.20)$. Note that by $(3.22)$ one has

$$
\frac{U_{T}^{i+1}(\boldsymbol{L})}{\varphi_{T}^{i+1}(\boldsymbol{L})}=\alpha_{t_{i+2}}^{i+1 \mid i}\left(\boldsymbol{L}^{(i)}\right) \cdots \alpha_{T}^{n \mid n-1}\left(\boldsymbol{L}^{(n)}\right)
$$

which implies

$$
\mathbb{E}^{\mathbb{Q}^{i+1}}\left[Y_{t_{i+2}}\left(\boldsymbol{L}^{(i+1)}\right) \varphi_{T}^{i+1}(\boldsymbol{L})^{-1} \mid \mathcal{G}_{t_{i+1}}^{i}\right]=J_{i+1}\left(Y_{t_{i+2}}\left(\boldsymbol{L}^{(i+1)}\right) \Phi_{t_{i+2}}\left(\boldsymbol{L}^{(i+1)}\right)\right) .
$$

In addition, Lemma 3.12 shows that

$$
\mathbb{E}^{\mathbb{Q}^{i+1}}\left[\varphi_{T}^{i+1}(\boldsymbol{L})^{-1} \mid \mathcal{G}_{t_{i+1}}^{i}\right]=J_{i+1}\left(\Phi_{t_{i+2}}\left(\boldsymbol{L}^{(i+1)}\right)\right)
$$

which implies (3.24) and completes the proof.

\section{Several stronger density hypotheses}

In this section, we consider particular cases of our successive density framework by introducing several density hypotheses stronger than Assumption 1. We compare these hypotheses and deduce concrete evaluation formulas in each case. For simplicity, we suppose that $\mathcal{F}_{0}$ is trivial.

\subsection{Density hypothesis with different initial $\sigma$-algebras}

In a first step we consider the conditional law of $L^{i}$ given the initial $\sigma$-algebra of the previous information filtration $\mathcal{G}_{0}^{i-1}=\sigma\left(\boldsymbol{L}^{(i-1)}\right)$.

Assumption 2 For any $i \in\{1, \cdots, n\}$, the $\mathcal{G}_{T}^{i-1}$-conditional law of $L^{i}$ is equivalent to its $\mathcal{G}_{0}^{i-1}$ conditional law under the probability $\mathbb{P}$, namely there exists a positive $\mathcal{G}_{T}^{i-1} \otimes \mathcal{E}$-measurable function $\beta_{T}^{i \mid i-1}\left(\boldsymbol{L}^{(i-1)}, \cdot\right)$ such that

$$
\mathbb{P}\left(L^{i} \in d x \mid \mathcal{G}_{T}^{i-1}\right)=\beta_{T}^{i \mid i-1}\left(\boldsymbol{L}^{(i-1)}, x\right) \mathbb{P}\left(L^{i} \in d x \mid \mathcal{G}_{0}^{i-1}\right) \text { a.s.. }
$$

Similarly to what we have explained in Remark 3.1, we can consider the conditional density $\beta_{T}^{i \mid i-1}\left(\boldsymbol{L}^{(i-1)}, \cdot\right)$ as a positive $\left(\mathcal{F}_{T} \otimes \mathcal{E}^{\otimes(i-1)}\right) \otimes \mathcal{E}$-mesurable function $\beta_{T}^{i \mid i-1}(\cdot, \cdot)$ evaluated at $\boldsymbol{L}^{(i-1)}$. For any $t \in[0, T]$, let $\beta_{t}^{i \mid i-1}(\cdot, \cdot)$ be an $\left(\mathcal{F}_{t} \otimes \mathcal{E}^{\otimes(i-1)}\right) \otimes \mathcal{E}$-measurable function such that

$$
\beta_{t}^{i \mid i-1}\left(\boldsymbol{L}^{(i-1)}, x\right)=\mathbb{E}^{\mathbb{P}}\left[\beta_{T}^{i \mid i-1}\left(\boldsymbol{L}^{(i-1)}, x\right) \mid \mathcal{G}_{t}^{i-1}\right] .
$$


By Corollary 3.5 ,

$$
\mathbb{P}\left(L^{i} \in d x \mid \mathcal{G}_{t}^{i-1}\right)=\beta_{t}^{i \mid i-1}\left(\boldsymbol{L}^{(i-1)}, x\right) \mathbb{P}\left(L^{i} \in d x \mid \mathcal{G}_{0}^{i-1}\right) .
$$

Note that $\beta_{0}^{i \mid i-1}\left(\boldsymbol{L}^{(i-1)}, x\right)=1$ a.s. for all $x \in E$ and

$$
\int_{E} \beta_{t}^{i \mid i-1}\left(\boldsymbol{L}^{(i-1)}, x\right) \mathbb{P}\left(L^{i} \in d x \mid \mathcal{G}_{0}^{i-1}\right)=1 .
$$

In particular, if we define for all $t_{i} \leq t \leq T$ a function $\alpha_{t}^{i \mid i-1}(\boldsymbol{x})$ on $\Omega \times E^{i}$ which is $\mathcal{F}_{T} \otimes \mathcal{E}^{i}$ measurable such that

$$
\alpha_{t}^{i \mid i-1}\left(\boldsymbol{x}^{(i)}\right)=\frac{\beta_{t}^{i \mid i-1}\left(\boldsymbol{x}^{(i)}\right)}{\beta_{t_{i}}^{i \mid i-1}\left(\boldsymbol{x}^{(i)}\right)}
$$

then the random vector $\boldsymbol{L}$ verifies Assumption 1 with the conditionnal density $\alpha_{T}^{i \mid i-1}\left(\boldsymbol{L}^{(i-1)}, x\right)$ and $\alpha_{t}^{i \mid i-1}\left(\boldsymbol{L}^{(i-1)}, x\right)=\mathbb{E}^{\mathbb{P}}\left[\alpha_{T}^{i \mid i-1}\left(\boldsymbol{L}^{(i-1)}, x\right) \mid \mathcal{G}_{t}^{i-1}\right], x \in E$.

Let us notice that under Assumption 2 the filtration $\mathbb{G}^{i}$ is right-continuous on $[0, T]$, whereas it is a priori right-continuous only on $\left[t_{i}, T\right]$ under the weaker Assumption 1.

We now apply Theorem 3.13 to compute the conditional expectation under Assumption 2 where the recursive operators can be simplified in an explicit manner. As the result can also be obtained in a more straightforward manner using a global approach (see Subsection 4.2), we will give the proof by using the recursive approach in the Appendix 6.1.

Proposition 4.1 We assume Assumption 2, Let $Y_{T}(\boldsymbol{L})$ be a non-negative $\mathcal{G}_{T}^{n}$-measurable random variable. Then for $t \in[0, T]$ one has

$\mathbb{E}^{\mathbb{P}}\left[Y_{T}(\boldsymbol{L}) \mid \mathcal{G}_{t}^{I}\right]=\left.\sum_{i=1}^{n} 1_{\left[t_{i}, t_{i+1}\right)}(t) \int_{E^{n-i}} \frac{\mathbb{E}^{\mathbb{P}}\left[Y_{T}(\boldsymbol{x}) Z_{T}^{n}(\boldsymbol{x}) \mid \mathcal{F}_{t}\right]}{Z_{t}^{i}\left(\boldsymbol{x}^{(i)}\right)}\right|_{\boldsymbol{x}^{(i)}=\boldsymbol{L}^{(i)}} \mathbb{P}\left(L^{i+1} \in d x^{i+1}, \cdots, L^{n} \in d x^{n} \mid \mathcal{G}_{0}^{i}\right)$

where the pricing kernel is defined as

$$
Z_{t}^{i}\left(\boldsymbol{x}^{(i)}\right):=\prod_{k=1}^{i} \beta_{t}^{k \mid k-1}\left(\boldsymbol{x}^{(k)}\right)
$$

We give the following key property of the pricing kernel.

Lemma 4.2 For $i \in\{0, \ldots, n-1\}$ and $t \in[0, T]$,

$$
\mathbb{P}\left(L^{i+1} \in d x^{i+1}, \cdots, L^{n} \in d x^{n} \mid \mathcal{G}_{t}^{i}\right)=\frac{Z_{t}^{n}\left(\boldsymbol{L}^{(i)}, x^{i+1}, \cdots, x^{n}\right)}{Z_{t}^{i}\left(\boldsymbol{L}^{(i)}\right)} \mathbb{P}\left(L^{i+1} \in d x^{i+1}, \cdots, L^{n} \in d x^{n} \mid \mathcal{G}_{0}^{i}\right)
$$

with convention $Z_{t}^{0}=1$. Moreover, one has

$$
Z_{t}^{i}\left(\boldsymbol{L}^{(i)}\right)=\int_{E^{n-i}} Z_{t}^{n}\left(\boldsymbol{L}^{(i)}, x^{i+1}, \cdots, x^{n}\right) \mathbb{P}\left(L^{i+1} \in d x^{i+1}, \cdots, L^{n} \in d x^{n} \mid \mathcal{G}_{0}^{i}\right) .
$$


ProOF: Let $I_{t}^{i}$ be the operator sending a non-negative $\mathcal{G}_{t}^{i}$-measurable random variable $Y_{t}\left(\boldsymbol{L}^{(i)}\right)$ to

$$
\mathbb{E}\left[Y_{t}\left(\boldsymbol{L}^{(i)}\right) \mid \mathcal{G}_{t}^{i-1}\right]=\int_{E} Y_{t}\left(\boldsymbol{L}^{(i-1)}, x^{i}\right) \beta_{t}^{i \mid i-1}\left(\boldsymbol{L}^{(i-1)}, x^{i}\right) \mathbb{P}\left(L^{i} \in d x^{i} \mid \mathcal{G}_{0}^{i-1}\right) .
$$

On the one hand, by the property of conditional expectation, one has,

$$
\begin{aligned}
\left(I_{t}^{i+1} \circ \cdots \circ I_{t}^{n}\right) & \left(Y_{t}(\boldsymbol{L})\right)=\mathbb{E}^{\mathbb{P}}\left[Y_{t}(\boldsymbol{L}) \mid \mathcal{G}_{t}^{i}\right] \\
& =\int_{E^{n-i}} Y_{t}\left(\boldsymbol{L}^{(i)}, x^{i+1}, \cdots, x^{n}\right) \mathbb{P}\left(L^{i+1} \in d x^{i+1}, \cdots, L^{n} \in d x^{n} \mid \mathcal{G}_{t}^{i}\right) .
\end{aligned}
$$

On the other hand, by the definition of the operators $I_{t}^{i+1}, \cdots, I_{t}^{n}$ and the fact

$$
\beta^{i+1 \mid i}\left(\boldsymbol{L}^{(i)}, x^{i+1}\right) \cdots \beta^{n \mid n-1}\left(\boldsymbol{L}^{(i)}, x^{i+1}, \cdots, x^{n}\right)=\frac{Z_{t}^{n}\left(\boldsymbol{L}^{(i)}, x^{i+1}, \cdots, x^{n}\right)}{Z_{t}^{i}\left(\boldsymbol{L}^{(i)}\right)},
$$

it follows

$$
\begin{aligned}
& \left(I_{t}^{i+1} \circ \cdots \circ I_{t}^{n}\right)\left(Y_{t}(\boldsymbol{L})\right) \\
= & \int_{E^{n-i}} Y_{t}\left(\boldsymbol{L}^{(i)}, x^{i+1}, \cdots, x^{n}\right) \frac{Z_{t}^{n}\left(\boldsymbol{L}^{(i)}, x^{i+1}, \cdots, x^{n}\right)}{Z_{t}^{i}\left(\boldsymbol{L}^{(i)}\right)} \mathbb{P}\left(L^{n} \in d x^{n} \mid \mathcal{G}_{0}^{n-1}\right) \cdots \mathbb{P}\left(L^{i+1} \in d x^{i+1} \mid \mathcal{G}_{0}^{i}\right) \\
= & \int_{E^{n-i}} Y_{t}\left(\boldsymbol{L}^{(i)}, x^{i+1}, \cdots, x^{n}\right) \frac{Z_{t}^{n}\left(\boldsymbol{L}^{(i)}, x^{i+1}, \cdots, x^{n}\right)}{Z_{t}^{i}\left(\boldsymbol{L}^{(i)}\right)} \mathbb{P}\left(L^{i+1} \in d x^{i+1}, \cdots, L^{n} \in d x^{n} \mid \mathcal{G}_{0}^{i}\right) .
\end{aligned}
$$

Combining with the equality (4.8), we deduce the first assertion (4.6) of the lemma, which leads to 4.7 directly.

Another hypothesis is the Jacod hypothesis in the successive initial enlargement of filtration setting where the terminal conditional law of each $L^{i}$ given the previous information filtration $\mathbb{G}^{(i-1)}$ is equivalent to its probability law.

Assumption 3 For any $i \in\{1, \cdots, n\}$, the $\mathcal{G}_{T}^{i-1}$-conditional law of $L^{i}$ is equivalent to its conditional law under the probability $\mathbb{P}$, namely there exists a positive $\mathcal{G}_{T}^{i-1} \otimes \mathcal{E}$-measurable function $p_{T}^{i \mid i-1}\left(\boldsymbol{L}^{(i-1)}, \cdot\right)$ such that

$$
\mathbb{P}\left(L^{i} \in d x \mid \mathcal{G}_{T}^{i-1}\right)=p_{T}^{i \mid i-1}\left(\boldsymbol{L}^{(i-1)}, x\right) \mathbb{P}\left(L^{i} \in d x\right) \text { a.s. }
$$

Note that, under the above assumption, for any $t \in[0, T]$, the $\mathcal{G}_{t}^{i-1}$-conditional law of $L^{i}$ has the density $p_{t}^{i \mid i-1}\left(\boldsymbol{L}^{(i-1)}, x\right):=\mathbb{E}^{\mathbb{P}}\left[p_{T}^{i \mid i-1}\left(\boldsymbol{L}^{(i-1)}, x\right) \mid \mathcal{G}_{t}^{i-1}\right]$ with respect to $\mathbb{P}\left(L^{i} \in d x\right)$. In particular, the family of $\left(\mathbb{P}, \mathbb{G}^{i-1}\right)$-martingales $p^{i \mid i-1}\left(\boldsymbol{L}^{(i-1)}, \cdot\right)$ has the initial value

$$
p_{0}^{i \mid i-1}\left(\boldsymbol{L}^{(i-1)}, x\right)=\frac{\mathbb{P}\left(L^{i} \in d x \mid \mathcal{G}_{0}^{i-1}\right)}{\mathbb{P}\left(L^{i} \in d x\right)}, \quad x \in E .
$$


Moreover, if $\boldsymbol{L}$ satisfies Assumption 3 it also satisfies Assumption 2 with $\beta_{T}^{i \mid i-1}\left(\boldsymbol{L}^{(i-1)}, x\right)$ where for all $t$,

$$
\beta_{t}^{i \mid i-1}\left(\boldsymbol{x}^{(i)}\right)=\frac{p_{t}^{i \mid i-1}\left(\boldsymbol{x}^{(i)}\right)}{p_{0}^{i \mid i-1}\left(\boldsymbol{x}^{(i)}\right)}
$$

We give hereafter an example where Assumption 3 is satisfied and the density processes $p^{i \mid i-1}$ are given explicitly.

Example 4.3 Let $\left(W, W^{\prime}\right)$ a two-dimensional Brownian motion and $\mathcal{F}_{t}=\sigma\left(W_{s}, s \leq t \leq T\right)$. Define the Brownian motion $B=\rho W+(1-\rho) W^{\prime}$ with $\rho \in\left[0,1\left[\right.\right.$ and let $L^{i}=B_{t_{i+1}}$ be the endpoint of $B$ at each interval $\left[t_{i}, t_{i+1}[\right.$. Then Assumption 3 is satisfied and

$$
p_{t}^{i \mid i-1}\left(L^{(i-1)}, x\right)=\left\{\begin{array}{l}
\frac{\phi\left(L^{i-1}, t_{i+1}-t_{i}, x\right)}{\phi\left(0, t_{i+1}, x\right)}, t \leq t_{i} \\
\frac{\phi\left(L^{i-1}+\rho\left(W_{t}-W_{t_{i}}\right), \rho^{2}\left(t_{i+1}-t\right)+(1-\rho)^{2}\left(t_{i+1}-t_{i}\right), x\right)}{\phi\left(0, t_{i+1}, x\right)}, t_{i}<t \leq t_{i+1} \\
\frac{\phi\left(L^{i-1}+\rho\left(W_{t_{i+1}}-W_{t_{i}}\right),(1-\rho)^{2}\left(t_{i+1}-t_{i}\right), x\right)}{\phi\left(0, t_{i+1}, x\right)}, t_{i+1}<t \leq T
\end{array}\right.
$$

where $\phi\left(\mu, \sigma^{2}, x\right)$ is the probability density function of the normal distribution $N\left(\mu, \sigma^{2}\right)$. We note that $p^{i \mid i-1}\left(\boldsymbol{L}^{(i-1)}, x\right)$ is a $\left(\mathbb{P}, \mathbb{G}^{i-1}\right)$-martingale on $[0, \mathrm{~T}]$.

We deduce from Proposition 4.1 the following result.

Proposition 4.4 We assume Assumption 3, Let $Y_{T}(\boldsymbol{L})$ be a non-negative $\mathcal{G}_{T}^{n}$-measurable random variable. Then for $t \in[0, T]$ one has

$\mathbb{E}^{\mathbb{P}}\left[Y_{T}(\boldsymbol{L}) \mid \mathcal{G}_{t}^{I}\right]=\left.\sum_{i=1}^{n} 1_{\left[t_{i}, t_{i+1}\right)}(t) \int_{E^{n-i}} \frac{\mathbb{E}^{\mathbb{P}}\left[Y_{T}(\boldsymbol{x}) \widetilde{Z}_{T}^{n}(\boldsymbol{x}) \mid \mathcal{F}_{t}\right]}{\widetilde{Z}_{t}^{i}\left(\boldsymbol{x}^{(i)}\right)}\right|_{\boldsymbol{x}^{(i)}=\boldsymbol{L}^{(i)}} \mathbb{P}\left(L^{i+1} \in d x^{i+1}\right) \cdots \mathbb{P}\left(L^{n} \in d x^{n}\right)$

where

$$
\widetilde{Z}_{t}^{i}\left(\boldsymbol{x}^{(i)}\right)=\prod_{k=1}^{i} p_{t}^{k \mid k-1}\left(\boldsymbol{x}^{(k)}\right) .
$$

Proof: We apply Proposition 4.1 under Assumption 3, By the equality 4.11) we obtain

$$
Z_{t}^{i}\left(\boldsymbol{x}^{(i)}\right)=\prod_{k=1}^{i} \beta_{t}^{k \mid k-1}\left(\boldsymbol{x}^{(k)}\right)=\widetilde{Z}_{t}^{i}\left(\boldsymbol{x}^{(i)}\right) \prod_{k=1}^{i} \frac{1}{p_{0}^{k \mid k-1}\left(\boldsymbol{x}^{(k)}\right)} .
$$

Therefore Proposition 4.1 leads to

$$
\begin{aligned}
\mathbb{E}^{\mathbb{P}}\left[Y_{T}(\boldsymbol{L}) \mid \mathcal{G}_{t}^{I}\right]=\sum_{i=1}^{n} 1_{\left[t_{i}, t_{i+1}\right)}(t) & \left.\int_{E^{n-i}} \frac{\mathbb{E}^{\mathbb{P}}\left[Y_{T}(\boldsymbol{x}) \widetilde{Z}_{T}^{n}(\boldsymbol{x}) \mid \mathcal{F}_{t}\right]}{\widetilde{Z}_{t}^{i}\left(\boldsymbol{x}^{(i)}\right)}\right|_{\boldsymbol{x}^{(i)}=\boldsymbol{L}^{(i)}} \\
& \prod_{k=i+1}^{n} \frac{1}{p_{0}^{k \mid k-1}\left(\boldsymbol{x}^{(k)}\right)} \mathbb{P}\left(L^{i+1} \in d x^{i+1}, \cdots, L^{n} \in d x^{n} \mid \mathcal{G}_{0}^{i}\right) .
\end{aligned}
$$


Finally, by (4.10) which implies the following relation

$$
\mathbb{P}\left(L^{i+1} \in d x^{i+1}, \cdots, L^{n} \in d x^{n} \mid \mathcal{G}_{0}^{i}\right)=\left(\prod_{k=i+1}^{n} p_{0}^{k \mid k-1}\left(\boldsymbol{x}^{(k)}\right)\right) \mathbb{P}\left(L^{i+1} \in d x^{i+1}\right) \cdots \mathbb{P}\left(L^{n} \in d x^{n}\right),
$$

we obtain the result of the proposition.

Remark 4.5 Similarly to Lemma 4.2 , for $i \in\{0, \cdots, n-1\}$ and $t \in[0, T]$, one has

$$
\mathbb{P}\left(L^{i+1} \in d x^{i+1}, \cdots, L^{n} \in d x^{n} \mid \mathcal{G}_{t}^{i}\right)=\frac{\widetilde{Z}_{t}^{n}\left(\boldsymbol{L}^{(i)}, x^{i+1}, \cdots, x^{n}\right)}{\widetilde{Z}_{t}^{i}\left(\boldsymbol{L}^{(i)}\right)} \mathbb{P}\left(L^{i+1} \in d x^{i+1}\right) \cdots \mathbb{P}\left(L^{n} \in d x^{n}\right),
$$

where $\widetilde{Z}_{t}^{0}=1$ and

$$
\widetilde{Z}_{t}^{i}\left(\boldsymbol{L}^{(i)}\right)=\int_{E^{n-i}} \widetilde{Z}_{t}^{n}\left(\boldsymbol{L}^{(i)}, x^{i+1}, \cdots, x^{n}\right) \mathbb{P}\left(L^{i+1} \in d x^{i+1}\right) \cdots \mathbb{P}\left(L^{n} \in d x^{n}\right) .
$$

Due to the transitivity of the equivalence relation between probability measures,

$$
\text { Assumption } 3 \Rightarrow \text { Assumption } 2 \Rightarrow \text { Assumption } 1 \text {. }
$$

We now provide several examples to compare these hypotheses.

Example 4.6 (1) Trivial examples (that lead to no enlargement of filtrations) show that the reciprocal statements are false: for example, $L^{i}$ which is a deterministic function of $\boldsymbol{L}^{(i-1)}$ satisfies Assumption 2 but not Assumption 3: $L_{i}$, which is a $\mathcal{G}_{t_{i}}^{i-1}$-measurable random variable but not $\mathcal{G}_{0}^{i-1}$-measurable satisfies Assumption 1 and not Assumption 2

(2) More generally, Assumption 2 is satisfied but not Assumption 3 at step $t_{i}$ if and only if the distribution of $L^{i}$ is not equivalent to the conditional distribution of $L^{i}$ given $\boldsymbol{L}^{(i-1)}$.

(3) Here is another example, in the context of credit risk and default threshold, in which Assumption 2 is satisfied and not Assumption 3 . Let $L^{i}$ take two values $a$ or $b, a<b$. At time $t_{i}$, the manager has an anticipation of the firm value $X_{T^{\prime}+t_{i}}$ with $T^{\prime}>T$ and knows if this value will be above or below the constant target $c, X$ being an $\mathbb{F}$-adapted process. If $X_{T^{\prime}+t_{i+1}}$ is above the target and the former threshold $L^{i}$ was low, then the manager keep fixing a low level for the threshold $L^{i+1}$, otherwise she will fix a high level for $L^{i+1}$, i.e.

$$
L^{i+1}=a 1_{\left\{X_{T^{\prime}+t_{i+1}}>c\right\}} 1_{\left\{L^{i}=a\right\}}+b\left(1_{\left\{X_{T^{\prime}+t_{i+1}} \leq c\right\}}+1_{\left\{X_{T^{\prime}+t_{i+1}}>c\right\}} 1_{\left\{L^{i}=b\right\}}\right)
$$

In this example, the distribution of $L^{i+1}$ has two atoms $a$ and $b$ with positive probability, while the distribution of $L^{i+1}$ given the event $\left\{L^{i}=b\right\}$ is a Dirac measure.

(4) In the same line, here is an example in which Assumption 1 is satisfied but not Assumption 2. If $X_{T^{\prime}+t_{i+1}}$ and the current value $X_{t_{i+1}}$ is above the target $c$ then the manager keeps fixing a low level for the threshold $L^{i+1}$, otherwise she fixes a high level for $L^{i+1}$ :

$$
L^{i+1}=a 1_{\left\{X_{T^{\prime}+t_{i+1}}>c\right\}} 1_{\left\{X_{t_{i+1}}>c\right\}}+b\left(1_{\left\{X_{T^{\prime}+t_{i+1}} \leq c\right\}}+1_{\left\{X_{T^{\prime}+t_{i+1}}>c\right\}} 1_{\left\{X_{t_{i+1}} \leq c\right\}}\right)
$$


In this example, the distribution of $L^{i+1}$ (given $\boldsymbol{L}^{(i)}$ ) has two atoms $a$ and $b$ with positive probability, while the distribution of $L^{i+1}$ given the event $\left\{X_{t_{i+1}} \leq c\right\}$ is a Dirac measure.

As previously in Proposition 3.11, we can introduce a family of probability measures which satisfy the following properties.

Proposition 4.7 Under Assumption 2 (resp. Assumption 3), there exists a family of equivalent probability measures $\left\{\overline{\mathbb{Q}}^{i}, i=1, \cdots, n\right\}$ such that

1) $\overline{\mathbb{Q}}^{i}$ is identical to $\mathbb{P}$ on $\mathcal{G}_{T}^{i-1}$,

2) any $L^{k}, k \in\{1, \cdots, n\}$, has the same conditional law given $\mathcal{G}_{0}^{k-1}$ (resp. the same probability law) under $\overline{\mathbb{Q}}^{i}$ and $\mathbb{P}$,

3) under $\overline{\mathbb{Q}}^{i}$, the vector $\left(L^{i}, \cdots, L^{n}\right)$ and $\mathcal{G}_{T}^{i-1}$ are conditionally independent given $\mathcal{G}_{0}^{k-1}$ (resp. independent).

Moreover, the Radon-Nikodym derivative is given by

$$
\left.\frac{d \overline{\mathbb{Q}}^{k}}{d \mathbb{P}}\right|_{\mathcal{G}_{T}^{n}}=\prod_{i=k}^{n} \frac{1}{\beta_{T}^{i \mid i-1}\left(\boldsymbol{L}^{(i)}\right)}=\frac{Z_{T}^{k-1}\left(\boldsymbol{L}^{(k-1)}\right)}{Z_{T}^{n}(\boldsymbol{L})} \quad\left(\text { resp. } \prod_{i=k}^{n} \frac{1}{p_{T}^{i \mid i-1}\left(\boldsymbol{L}^{(i)}\right)}=\frac{\widetilde{Z}_{T}^{k-1}\left(\boldsymbol{L}^{(k-1)}\right)}{\widetilde{Z}_{T}^{n}(\boldsymbol{L})}\right) .
$$

\subsection{Global enlargement of filtration}

In this subsection, instead of assuming the density hypothesis in a successive way for the family of enlarged filtrations, we consider the random variables $L^{1}, \cdots, L^{n}$ as a vector and suppose the Jacod's hypothesis in the following way.

Assumption 4 The $\mathbb{F}$-conditional law of $\boldsymbol{L}=\left(L^{1}, \cdots, L^{n}\right)$ is equivalent to its probability law, i.e., there exists an $\mathcal{F}_{T} \otimes \mathcal{E}^{n}$-measurable function $p_{T}(\cdot)$ such that

$$
\mathbb{P}\left(\boldsymbol{L} \in d \boldsymbol{x} \mid \mathcal{F}_{t}\right)=p_{T}(\boldsymbol{x}) \mathbb{P}(\boldsymbol{L} \in d \boldsymbol{x}) \text { a.s. }
$$

where $d \boldsymbol{x}=\left(d x^{1}, \cdots, d x^{n}\right)$.

We denote by $\left(p_{t}(\boldsymbol{x}), t \in[0, T]\right)$ the density process of $\boldsymbol{L}$ given $\mathbb{F}$, which is a $(\mathbb{P}, \mathbb{F})$-martingale for any $\boldsymbol{x} \in E^{n}$. Define the filtration $\mathbb{G}^{\boldsymbol{L}}=\left(\mathcal{G}_{t}^{\boldsymbol{L}}\right)_{t \in[0, T]}$, where $\mathcal{G}_{t}^{\boldsymbol{L}}:=\mathcal{F}_{t} \vee \sigma(\boldsymbol{L})$ coincides with $\mathcal{G}_{t}^{n}$. Then, $\boldsymbol{L}$ and $\mathbb{F}$ are independent under the equivalent probability measure $\mathbb{P}^{L}$ defined by

$$
\left.\frac{d \mathbb{P}^{L}}{d \mathbb{P}}\right|_{\mathcal{G}_{t}^{L}}:=\frac{1}{p_{t}(\boldsymbol{L})} .
$$

Remark that $L^{1}, \cdots, L^{n}$ are not mutually independent under $\mathbb{P}^{L}$. In particular, if $\boldsymbol{L}$ is independent of $\mathcal{F}_{T}$, then $p_{t}(\boldsymbol{L})=1$.

We make precise the relationship between the global approach and the successive one. In particular, we compare Assumption 4 with previous assumptions. 
Proposition 4.8 1) Assumption 4 is equivalent to Assumption 2. The conditional densities are given by the following relations: on one hand,

$$
p_{T}(\boldsymbol{x})=\prod_{i=1}^{n} \beta_{T}^{i \mid i-1}\left(\boldsymbol{x}^{(i)}\right)
$$

and on the other hand,

$$
\beta_{T}^{i \mid i-1}\left(\boldsymbol{L}^{(i-1)}, x^{i}\right)=\frac{\int_{E^{n-i}} p_{T}\left(\boldsymbol{L}^{(i-1)}, x^{i}, \cdots, x_{n}\right) \mathbb{P}\left(L^{i+1} \in d x^{i+1}, \cdots, L^{n} \in d x^{n} \mid \mathcal{G}_{0}^{i}\right)}{\int_{E^{n-i+1}} p_{T}\left(\boldsymbol{L}^{(i-1)}, x^{i}, \cdots, x_{n}\right) \mathbb{P}\left(L^{i} \in d x^{i}, \cdots, L^{n} \in d x^{n} \mid \mathcal{G}_{0}^{i-1}\right)} .
$$

2) The probability measure $\mathbb{P}^{L}$ coincides with the probability measure $\overline{\mathbb{Q}}^{1}$ constructed in Proposition 4.7 under Assumption 2 .

Proof: If Assumption 2 holds, let $i=0$ in Lemma 4.2, we obtain

$$
\mathbb{P}\left(\boldsymbol{L} \in d \boldsymbol{x} \mid \mathcal{F}_{t}\right)=Z_{t}^{n}(\boldsymbol{x}) \mathbb{P}(\boldsymbol{L} \in d \boldsymbol{x}),
$$

which implies Assumption 4 with

$$
p_{t}(\boldsymbol{x})=Z_{t}^{n}(\boldsymbol{x}) .
$$

Moreover, by Proposition 4.7, $\mathbb{P}^{L}=\overline{\mathbb{Q}}^{1}$, which is the second assertion of the proposition.

Conversely, supposing Assumption $4, \mathbb{F}$ and $\boldsymbol{L}$ are independent under $\mathbb{P}^{L}$, thus for $i=1, \cdots, n$,

$$
\mathbb{P}^{L}\left(L^{i} \in d x^{i} \mid \mathcal{F}_{T} \vee \sigma\left(\boldsymbol{L}^{(i-1)}\right)\right)=\mathbb{P}^{L}\left(L^{i} \in d x^{i} \mid \boldsymbol{L}^{(i-1)}\right), \mathbb{P}-\text { a.s. }
$$

and we conclude, using the stability of Assumption 2 under an equivalent change of probability measure $\left(\mathbb{P}^{L}\right.$ is equivalent to $\left.\mathbb{P}\right)$, that

$$
\mathbb{P}\left(L^{i} \in d x^{i} \mid \mathcal{G}_{T}^{i-1}\right)(\omega) \sim \mathbb{P}\left(L^{i} \in d x^{i} \mid \mathcal{G}_{0}^{i-1}\right) .
$$

Moreover, the Radon-Nikodym density $d \mathbb{P} / d \mathbb{P}^{L}$ on $\mathcal{G}_{T}^{i}$ is given by

$$
Q_{T}^{i}\left(\boldsymbol{L}^{(i)}\right):=\mathbb{E}^{\mathbb{P}^{L}}\left[p_{T}(\boldsymbol{L}) \mid \mathcal{G}_{T}^{i}\right]=\int_{E^{n-i}} p_{T}\left(\boldsymbol{L}^{(i)}, x^{i+1}, \cdots, x^{n}\right) \mathbb{P}\left(L^{i+1} \in d x^{i+1}, \cdots, L^{n} \in d x^{n} \mid \mathcal{G}_{0}^{i}\right)
$$

since $\boldsymbol{L}$ and $\mathbb{F}$ are independent under $\mathbb{P}^{L}$ and $\mathbb{P}^{L}$ coincides with $\mathbb{P}$ on $\sigma(\boldsymbol{L})$. Therefore, by Remark 3.3 . we obtain that

$$
\mathbb{P}\left(L^{i} \in d x^{i} \mid \mathcal{G}_{T}^{i-1}\right)=\frac{Q_{T}^{i}\left(\boldsymbol{L}^{(i-1)}, x^{i}\right)}{\int_{E} Q_{T}^{i}\left(\boldsymbol{L}^{(i-1)}, x^{i}\right) \mathbb{P}\left(L^{i} \in d x^{i} \mid \mathcal{G}_{0}^{i-1}\right)} \mathbb{P}\left(L^{i} \in d x^{i} \mid \mathcal{G}_{0}^{i-1}\right),
$$

which leads to 4.17. 
Proposition 4.9 1) Assumption $\sqrt{4}$ together with the condition $\mathbb{P}(\boldsymbol{L} \in d \boldsymbol{x}) \sim \prod_{i=0}^{n} \mathbb{P}\left(L^{i} \in d x_{i}\right)$ is equivalent to Assumption 3. The conditional densities are given by the following relations: on one hand,

$$
p_{T}(\boldsymbol{x})=\frac{\widetilde{Z}_{T}^{n}(\boldsymbol{x})}{\widetilde{Z}_{0}^{n}(\boldsymbol{x})}=\prod_{i=1}^{n} \frac{p_{T}^{i \mid i-1}\left(\boldsymbol{x}^{(i)}\right)}{p_{0}^{i \mid i-1}\left(\boldsymbol{x}^{(i)}\right)}
$$

and on the other hand,

$$
p_{T}^{i \mid i-1}\left(\boldsymbol{L}^{(i-1)}, x^{i}\right)=\frac{\int_{E^{n-i}} \frac{p_{T}}{\zeta}\left(\boldsymbol{L}^{(i-1)}, x^{i}, \cdots, x^{n}\right) \mathbb{P}\left(L^{i+1} \in d x^{i+1}\right) \cdots \mathbb{P}\left(L^{n} \in d x^{n}\right)}{\int_{E^{n-i+1}} \frac{p_{T}}{\zeta}\left(\boldsymbol{L}^{(i-1)}, x^{i}, \cdots, x^{n}\right) \mathbb{P}\left(L^{i} \in d x^{i}\right) \cdots \mathbb{P}\left(L^{n} \in d x^{n}\right)},
$$

where $\zeta(\cdot)$ is the Radon-Nikodym density of $\prod_{i=1}^{n} \mathbb{P}\left(L^{i} \in d x^{i}\right)$ with respect to $\mathbb{P}(\boldsymbol{L} \in d \boldsymbol{x})$.

2) Under Assumption 4 and assuming $\mathbb{P}(\boldsymbol{L} \in d \boldsymbol{x})=\zeta(\boldsymbol{x})^{-1} \prod_{i=1}^{n} \mathbb{P}\left(L^{i} \in d x^{i}\right)$ with $\zeta(\cdot)$ being a positive function on $E^{n}$, the equivalent probability measure $\mathbb{Q}^{L}$ defined by

$$
\left.\frac{d \mathbb{Q}^{L}}{d \mathbb{P}}\right|_{\mathcal{G}_{T}^{n}}=\frac{\zeta(\boldsymbol{L})}{p_{T}(\boldsymbol{L})}
$$

satisfies

(a) $\mathbb{F}$ and the random variables $L^{1}, \cdots, L^{n}$ are mutually independent under $\mathbb{Q}^{L}$

(b) the marginal law of each $L_{1}, \cdots, L_{n}$ under $\mathbb{Q}^{L}$ coincide with the one under $\mathbb{P}$.

3) The probability measure $\mathbb{Q}^{L}$ coincides with the probability $\overline{\mathbb{Q}}^{1}$ defined in Proposition 4.7 under Assumption 3 .

Proof: 1) and 2) Under Assumption 3, by Remark 4.5 and taking $i=0$ in (4.13), we have

$$
\mathbb{P}\left(\boldsymbol{L} \in \boldsymbol{x} \mid \mathcal{F}_{T}\right)=\widetilde{Z}_{T}^{n}(\boldsymbol{x}) \mathbb{P}\left(L^{1} \in d x^{1}\right) \cdots \mathbb{P}\left(L^{n} \in d x^{n}\right)
$$

and in particular

$$
\mathbb{P}(\boldsymbol{L} \in \boldsymbol{x})=\widetilde{Z}_{0}^{n}(\boldsymbol{x}) \mathbb{P}\left(L^{1} \in d x^{1}\right) \cdots \mathbb{P}\left(L^{n} \in d x^{n}\right) .
$$

Therefore, Assumption 4 is true with $p_{T}(\boldsymbol{x})=\widetilde{Z}_{T}^{n}(\boldsymbol{x}) / \widetilde{Z}_{0}^{n}(\boldsymbol{x})$.

Conversely, we assume Assumption 4 and the condition $\mathbb{P}(\boldsymbol{L} \in \boldsymbol{x}) \sim \prod_{i=1}^{n} \mathbb{P}\left(L^{i} \in d x^{i}\right)$, with

$$
\mathbb{P}(\boldsymbol{L} \in \boldsymbol{x})=\zeta(\boldsymbol{x})^{-1} \prod_{i=1}^{n} \mathbb{P}\left(L^{i} \in d x^{i}\right)
$$

Note that the Assumption 4 implies the existence of a probability measure $\mathbb{P}^{L}$ equivalent to $\mathbb{P}$ such that $\boldsymbol{L}$ is independent of $\mathcal{F}_{T}$ under $\mathbb{P}^{L}$ and that $\mathbb{P}^{L}$ coincides with $\mathbb{P}$ on $\mathcal{F}_{T}$ and on $\sigma(L)$. Therefore

$$
\mathbb{P}^{L}(\boldsymbol{L} \in d \boldsymbol{x})=\mathbb{P}(\boldsymbol{L} \in d \boldsymbol{x})=\zeta(\boldsymbol{x})^{-1} \prod_{i=1}^{n} \mathbb{P}\left(L^{i} \in d x^{i}\right)=\zeta(\boldsymbol{x})^{-1} \prod_{i=1}^{n} \mathbb{P}^{L}\left(L^{i} \in d x^{i}\right),
$$


which implies that

$$
\mathbb{E}^{\mathbb{P}^{L}}[\zeta(\boldsymbol{L})]=\int_{E^{n}} \frac{\zeta(\boldsymbol{x})}{\zeta(\boldsymbol{x})} \prod_{i=1}^{n} \mathbb{P}^{L}\left(L^{i} \in d x^{i}\right)=1 .
$$

We introduce a new probability measure $\mathbb{Q}^{L}$ on $\mathcal{G}_{T}^{\boldsymbol{L}}$ such that $d \mathbb{Q}^{L} / d \mathbb{P}^{L}=\zeta(\boldsymbol{L})$, which is also given by (4.21). We then check (a) and (b) in the second assertion.

- We first prove that $\boldsymbol{L}$ and $\mathcal{F}_{T}$ are independent under $\mathbb{Q}^{L}$. Let $f$ be a bounded Borel function on $E^{n}$ and $X$ be a bounded $\mathcal{F}_{T}$-measurable random variable. One has

$$
\mathbb{E}^{\mathbb{Q}^{L}}[f(\boldsymbol{L}) \cdot X]=\mathbb{E}^{\mathbb{P}^{L}}[\zeta(\boldsymbol{L}) f(\boldsymbol{L}) X]=\mathbb{E}^{\mathbb{P}^{L}}[\zeta(\boldsymbol{L}) f(\boldsymbol{L})] \cdot \mathbb{E}^{\mathbb{P}^{L}}[X]=\mathbb{E}^{\mathbb{Q}^{L}}[f(\boldsymbol{L})] \mathbb{E}^{\mathbb{P}^{L}}[X],
$$

where the second equality comes from the fact that $\boldsymbol{L}$ and $\mathcal{F}_{T}$ are independent under $\mathbb{P}^{L}$. Taking $f=1$ in the last expression leads to

$$
\mathbb{E}^{\mathbb{P}^{L}}[X]=\mathbb{E}^{\mathbb{Q}^{L}}[X],
$$

therefore $\mathbb{E}^{\mathbb{Q}^{L}}[f(\boldsymbol{L}) X]=\mathbb{E}^{\mathbb{Q}^{L}}[f(\boldsymbol{L})] \cdot \mathbb{E}^{\mathbb{Q}^{L}}[X]$.

- Moreover, the random variables $L^{1}, \cdots, L^{n}$ are independent under $\mathbb{Q}^{L}$. Indeed, if $f_{1}, \cdots, f_{n}$ are bounded Borel functions on $E$, one has

$$
\begin{aligned}
& \mathbb{E}^{\mathbb{Q}^{L}}\left[f_{1}\left(L^{1}\right) \cdots f_{n}\left(L^{n}\right)\right]=\mathbb{E}^{\mathbb{P}^{L}}\left[\zeta(\boldsymbol{L}) f_{1}\left(L^{1}\right) \cdots f_{n}\left(L^{n}\right)\right] \\
= & \int_{E^{n}} \zeta(\boldsymbol{x}) f_{1}\left(x^{1}\right) \cdots f_{n}\left(x^{n}\right) \mathbb{P}^{L}(\boldsymbol{L} \in d \boldsymbol{x}) \\
= & \int_{E^{n}} f_{1}\left(x^{1}\right) \cdots f_{n}\left(x^{n}\right) \prod_{i=1}^{n} \mathbb{P}^{L}\left(L^{i} \in d x^{i}\right)=\prod_{i=1}^{n} \mathbb{E}^{\mathbb{P}^{L}}\left[f_{i}\left(L^{i}\right)\right] .
\end{aligned}
$$

Besides, taking $f_{j}=1$ for all $j \neq i$ gives

$$
\mathbb{E}^{\mathbb{Q}^{L}}\left[f_{i}\left(L^{i}\right)\right]=\mathbb{E}^{\mathbb{P} L}\left[f_{i}\left(L^{i}\right)\right]=\mathbb{E}^{\mathbb{P}}\left[f_{i}\left(L^{i}\right)\right] .
$$

Therefore

$$
\mathbb{E}^{\mathbb{Q}^{L}}\left[f_{1}\left(L^{1}\right) \cdots f_{n}\left(L^{n}\right)\right]=\prod_{i=1}^{n} \mathbb{E}^{\mathbb{Q}^{L}}\left[f_{i}\left(L^{i}\right)\right] .
$$

- The two previous points gives

$$
\mathbb{Q}^{L}\left(L^{i} \in d x^{i} \mid \mathcal{G}_{T}^{i-1}\right)=\mathbb{Q}^{L}\left(L^{i} \in d x^{i}\right) .
$$

Moreover, the Radon-Nikodym density $d \mathbb{P} / d \mathbb{Q}^{L}$ on $\mathcal{G}_{T}^{i}$ is given by

$$
\mathbb{E}^{\mathbb{Q}^{L}}\left[\frac{p_{T}(\boldsymbol{L})}{\zeta(\boldsymbol{L})} \mid \mathcal{G}_{T}^{i}\right]=\int_{E^{n-i}} \frac{p_{T}}{\zeta}\left(\boldsymbol{L}^{(i)}, x^{i+1}, \cdots, x_{n}\right) \mathbb{P}\left(L^{i+1} \in d x^{i+1}\right) \cdots \mathbb{P}\left(L^{n} \in d x^{n}\right) .
$$

By Remark 3.3, this implies Assumption 3 with

$$
p_{t}^{i \mid i-1}\left(\boldsymbol{L}^{(i-1)}, x\right)=\frac{\int_{E^{n-i}} \frac{p_{T}}{\zeta}\left(\boldsymbol{L}^{(i-1)}, x^{i}, \cdots, x^{n}\right) \mathbb{P}\left(L^{i+1} \in d x^{i+1}\right) \cdots \mathbb{P}\left(L^{n} \in d x^{n}\right)}{\int_{E^{n-i+1}} \frac{p_{T}}{\zeta}\left(\boldsymbol{L}^{(i-1)}, x^{i}, \cdots, x^{n}\right) \mathbb{P}\left(L^{i} \in d x^{i}\right) \cdots \mathbb{P}\left(L^{n} \in d x^{n}\right)} .
$$


Therefore the assertions 1) and 2) are proved.

3) Finally, to prove the third assertion, it suffices to verify that $\left(\prod_{i=1}^{n} p_{T}^{i \mid i-1}\left(\boldsymbol{L}^{(i)}\right)\right)^{-1}$ is equal to $\zeta(\boldsymbol{L}) / p_{T}(\boldsymbol{L})$. This is a consequence of 4.19 since 4.22 leads to

$$
\zeta(\boldsymbol{x})=\frac{1}{\widetilde{Z}_{0}^{n}(\boldsymbol{x})}=\prod_{i=1}^{n} \frac{1}{p_{0}^{i \mid i-1}\left(\boldsymbol{x}^{(i)}\right)} .
$$

The proposition is thus proved.

Remark 4.10 In the particular case where the law of $\boldsymbol{L}$ admits a density with respect to the Lebesgue measure on $E^{n}$, Assumptions 3 and 4 are equivalent.

Remark 4.11 The function $\zeta$ can be expressed in terms of copulas: let $c\left(u^{1}, \cdots, u^{n}\right)$ denotes the density of the copula such that

$$
C\left(u^{1}, \cdots, u^{n}\right)=F\left(F_{1}^{-1}\left(u^{1}\right), \cdots, F_{n}^{-1}\left(u^{n}\right)\right)=\int_{-\infty}^{u^{1}} \cdots \int_{-\infty}^{u^{n}} c\left(u^{1}, \cdots, u^{n}\right) d u^{1} \cdots d u^{n}
$$

where $F_{1}, \cdots, F_{n}$ are marginal distribution functions and $F$ is the joint distribution function, then

$$
\zeta\left(x^{1}, \cdots, x^{n}\right)=\frac{1}{c\left(F_{1}\left(x^{1}\right), \cdots, F_{n}\left(x^{n}\right)\right)}
$$

\subsection{Conditional expectation using the global approach}

We now apply the global approach to calculate the conditional expectations with respect to the insider's filtration $\mathbb{G}^{I}$, under the equivalent Assumptions 2 and 4 . The idea is to use the global change of probability measure $\mathbb{P}^{L}$, which will make easier the computation.

Proposition 4.12 We assume Assumption 4. Let $Y_{T}(\boldsymbol{L})$ be a non-negative $\mathcal{G}_{T}^{n}$-measurable random variable. Then, for $t \in[0, T]$,

$\mathbb{E}^{\mathbb{P}}\left[Y_{T}(\boldsymbol{L}) \mid \mathcal{G}_{t}^{I}\right]=\left.\sum_{i=1}^{n} 1_{\left[t_{i}, t_{i+1}\right)}(t) \frac{\int_{E^{n-i}} \mathbb{E}^{\mathbb{P}}\left[Y_{T}(\boldsymbol{x}) p_{T}(\boldsymbol{x}) \mid \mathcal{F}_{t}\right] \mathbb{P}\left(L^{i+1} \in d x^{i+1}, \cdots, L^{n} \in d x^{n} \mid \boldsymbol{L}^{(i)}\right)}{\int_{E^{n-i}} p_{t}(\boldsymbol{x}) \mathbb{P}\left(L^{i+1} \in d x^{i+1}, \cdots, L^{n} \in d x^{n} \mid \boldsymbol{L}^{(i)}\right)}\right|_{\boldsymbol{x}^{(i)}=\boldsymbol{L}^{(i)}}$

Proof: We use the change of probability measure to $\mathbb{P}^{L}$ constructed in the global approach Subsection 4.2. By Bayes formula, one has

$$
1_{\left[t_{i}, t_{i+1}\right)} \mathbb{E}^{\mathbb{P}}\left[Y_{T}(\boldsymbol{L}) \mid \mathcal{G}_{t}^{I}\right]=1_{\left[t_{i}, t_{i+1}\right)} \mathbb{E}^{\mathbb{P}}\left[Y_{T}(\boldsymbol{L}) \mid \mathcal{G}_{t}^{i}\right]=1_{\left[t_{i}, t_{i+1}\right)} \frac{\mathbb{E}^{\mathbb{P}^{L}}\left[\left(Y_{T} p_{T}\right)(\boldsymbol{L}) \mid \mathcal{G}_{t}^{i}\right]}{\mathbb{E}^{\mathbb{P}^{L}}\left[p_{T}(\boldsymbol{L}) \mid \mathcal{G}_{t}^{i}\right]}
$$


Since $\boldsymbol{L}$ and $\mathbb{F}$ are independent under $\mathbb{P}^{L}$, and $\mathbb{P}^{L}$ coincides with $\mathbb{P}$ on $\mathbb{F}$, and $\sigma(\boldsymbol{L})$ respectively, one has

$$
\begin{aligned}
& \mathbb{E}^{\mathbb{P}^{L}}\left[p_{T}\left(L^{1}, \cdots, L^{n}\right) \mid \mathcal{G}_{t}^{i}\right] \\
= & \left.\left(\int_{E^{n-i}} \mathbb{E}^{\mathbb{P}}\left[p_{T}\left(\boldsymbol{x}^{(i)}, x^{i+1}, \cdots, x^{n}\right) \mid \mathcal{F}_{t}\right] \mathbb{P}\left(L^{i+1} \in d x^{i+1}, \cdots, L^{n} \in d x^{n} \mid \boldsymbol{L}^{(i)}\right)\right)\right)\left.\right|_{\boldsymbol{x}^{(i)}=\boldsymbol{L}^{(i)}} \\
= & \left.\left(\int_{E^{n-i}} p_{t}\left(\boldsymbol{x}^{(i)}, x^{i+1}, \cdots, x^{n}\right) \mathbb{P}\left(L^{i+1} \in d x^{i+1}, \cdots, L^{n} \in d x^{n} \mid \boldsymbol{L}^{(i)}\right)\right)\right)\left.\right|_{\boldsymbol{x}^{(i)}=\boldsymbol{L}^{(i)}} .
\end{aligned}
$$

where the second equality results from the martingale property of $\left(p_{t}(\boldsymbol{x})\right)_{t \in[0, T]}$. Moreover,

$$
\begin{aligned}
& \mathbb{E}^{\mathbb{P}^{L}}\left[\left(Y_{T} p_{T}\right)\left(L^{1}, \cdots, L^{n}\right) \mid \mathcal{G}_{t}^{i}\right] \\
= & \left.\left(\int_{E^{n-i}} \mathbb{E}^{\mathbb{P}}\left[\left(Y_{T} p_{T}\right)\left(\boldsymbol{x}^{(i)}, x^{i+1}, \cdots, x^{n}\right) \mid \mathcal{F}_{t}\right] \mathbb{P}\left(L^{i+1} \in d x^{i+1}, \cdots, L^{n} \in d x^{n} \mid \boldsymbol{L}^{(i)}\right)\right)\right)\left.\right|_{\boldsymbol{x}^{(i)}=\boldsymbol{L}^{(i)}}
\end{aligned}
$$

which completes the proof.

Remark 4.13 By the equality $p_{T}(\boldsymbol{x})=Z_{T}^{n}(\boldsymbol{x})$ (c.f. (4.18)) and the relation (4.7), we see that Proposition 4.12 gives the same result as in Proposition 4.1 under Assumption 2.

Remark 4.14 If Assumption 3 is satisfied, then

$$
\mathbb{P}\left(L^{i+1} \in d x^{i+1}, \cdots, L^{n} \in d x^{n} \mid \boldsymbol{L}^{(i)}\right)=\frac{1}{\zeta\left(\boldsymbol{L}^{(i)}, x^{i+1}, \cdots, x^{n}\right)} \mathbb{P}\left(L^{i+1} \in d x^{i+1}\right) \cdots \mathbb{P}\left(L^{n} \in d x^{n}\right) .
$$

Then as a direct consequence of Proposition 4.12, we have

$$
\mathbb{E}^{\mathbb{P}}\left[Y_{T}(\boldsymbol{L}) \mid \mathcal{G}_{t}^{I}\right]=\left.\sum_{i=1}^{n} 1_{\left[t_{i}, t_{i+1}\right)}(t) \int_{E^{n-i}} \frac{\mathbb{E}^{\mathbb{P}}\left[\left(Y_{T} \frac{p_{T}}{\zeta}\right)\left(\boldsymbol{x}^{(i)}, x^{i+1}, \cdots, x^{n}\right) \mid \mathcal{F}_{t}\right]}{\frac{p_{t}}{\zeta}\left(\boldsymbol{x}^{(i)}, x^{i+1}, \cdots, x^{n}\right)}\right|_{\boldsymbol{x}^{(i)}=\boldsymbol{L}^{(i)}} \prod_{k=i+1}^{n} \mathbb{P}\left(L^{k} \in d x^{k}\right) .
$$

\section{Application and numerical illustration}

In this section, we apply our framework to a default model with insider's information. We are particularly interested in the default/survival probability and the pricing of defaultable bonds under different information levels.

We consider the default time of a firm which is supposed to be the first time that a continuous $\mathbb{F}$-adapted process $\left(X_{t}, t \geq 0\right)$ reaches a random threshold, which is determined by the manager of the firm and can be adjusted dynamically. More precisely, let the default threshold $\left(L_{t}, t \in[0, T]\right)$ be given in the form 2.2 . The default time is defined by

$$
\tau:=\inf \left\{t: X_{t}<L_{t}\right\}
$$

where the random variables $L^{1}, \cdots, L^{n}$ represent the private information of the manager on the threshold at times $t_{1}, \cdots, t_{n}$ which are not available by standard investors. This model extends 
the one considered in [14]. To make comparison with a standard investor, we also introduce the information filtration given by $\mathbb{G}=\left(\mathcal{G}_{t}\right)_{t \in[0, T]}$ where

$$
\mathcal{G}_{t}=\bigcap_{s \geq t} \mathcal{F}_{s} \vee \sigma(\tau \wedge s)
$$

The filtration $\mathbb{G}$ is the progressive enlargement of $\mathbb{F}$ by the random time $\tau$ and is classically used to model the available information in a default market for a standard agent, in comparison with the filtration $\mathbb{G}^{I}$ which represents the insider information.

\subsection{Conditional survival probability}

One of fundamental quantities in the modeling of credit risk is the conditional survival probability given the available information. The following result give the conditional survival probability given the insider information. For ease of computations, we suppose that Assumption 3 holds, but similar computations can be done under the other assumptions studied in this paper.

Proposition 5.1 Let $0 \leq t \leq s \leq T$. We denote by $i$ and $j$ the indexes such that $t_{i} \leq t<t_{i+1}$ and $t_{j} \leq s<t_{j+1}$. Then

$$
\mathbb{P}\left(\tau>s \mid \mathcal{G}_{t}^{I}\right)=\left.1_{\{\tau>t\}} \frac{\mathbb{E}^{\mathbb{P}}\left[\chi_{s}^{i}\left(\boldsymbol{x}^{(i)}\right) \mid \mathcal{F}_{t}\right]}{\int_{E^{n-i}} \frac{p_{s}}{\zeta}\left(\boldsymbol{x}^{(i)}, x^{i+1} \cdots, x^{n}\right) \prod_{k=i+1}^{n} \mathbb{P}\left(L^{k} \in d x^{k}\right)}\right|_{\boldsymbol{x}^{(i)}=\boldsymbol{L}^{(i)}} .
$$

where, denoting $X_{[t, s[}^{*}:=\inf _{t \leq u<s} X_{u}$ and $X_{t}^{*}:=X_{[0, t[}^{*}=\inf _{0 \leq u<t} X_{u}$, if $i<j$,

$$
\chi_{s}^{i}\left(\boldsymbol{x}^{(i)}\right)=\int_{E^{n-i}} \frac{p_{s}}{\zeta}(\boldsymbol{x}) 1_{\left\{X_{\left[t, t_{i+1} \mid\right.}^{*}>x^{i}\right\}} 1_{\left\{X_{\left[t_{i+1}, t_{i+2}[\right.}^{*}>x_{i+1}\right\}} \ldots 1_{\left\{X_{\left[t_{j}, s[\right.}^{*}>x_{j}\right\}} \prod_{k=i+1}^{n} \mathbb{P}\left(L^{k} \in d x^{k}\right) .
$$

and else if $i=j$

$$
\chi_{s}^{i}\left(\boldsymbol{x}^{(i)}\right)=\int_{E^{n-i}} \frac{p_{s}}{\zeta}(\boldsymbol{x}) 1_{\left\{X_{[t, s]}^{*}>x^{i}\right\}} \prod_{k=i+1}^{n} \mathbb{P}\left(L^{k} \in d x^{k}\right) .
$$

Proof: By the definitions (5.1) and 2.2), the survival event can be written as

$$
1_{\{\tau>s\}}=1_{\left\{X_{\left[t_{1}, t_{2}[\right.}^{*}>L^{1}\right\}} \cdots 1_{\left\{X_{\left[t_{i}, t\right]}^{*}>L^{i}\right\}} 1_{\left\{X_{\left[t, t_{i+1} \mid\right.}^{*}>L^{i}\right\}} \cdots 1_{\left\{X_{\left[t_{j}, s\right]}^{*}>L^{j}\right\}}
$$

We apply 4.26 to the random variable

$$
Y_{T}(\boldsymbol{x})=1_{\left\{X_{\left[t_{1}, t_{2}[\right.}^{*}>x^{1}\right\}} \cdots 1_{\left\{X_{\left[t_{i}, t\right]}^{*}>x^{i}\right\}} 1_{\left\{X_{\left[t, t_{i+1}[\right.}^{*}>x^{i}\right\}} \cdots 1_{\left\{X_{\left[t_{j}, s\right]}^{*}>x^{j}\right\}}
$$

and obtain the results.

We also recall that for the standard information, it is well known (see [5, 10]) that for $t \leq s$,

$$
\mathbb{P}\left(\tau>s \mid \mathcal{G}_{t}\right)=1_{\{\tau>t\}} \frac{\mathbb{P}\left(\tau>s \mid \mathcal{F}_{t}\right)}{\mathbb{P}\left(\tau>t \mid \mathcal{F}_{t}\right)} .
$$

In the following, we shall compare the survival probability estimated by these two types of investors in an explicit setting, in order to show the impact of insider information. 


\subsection{An explicit default model}

We consider now a concrete example with three periods $0=t_{1}<t_{2}<t_{3}=T$ where the firm value $X$ follows a geometric Brownian motion (with drift $\mu$ and volatility $\sigma$ ). The default threshold information are renewed at $t_{1}$ and $t_{2}$ respectively as $L^{1}$ and $L^{2}$ and we suppose that $L^{1}$ and $L^{2}$ are exponential random variables with intensity $\lambda_{1}$ and $\lambda_{2}$ respectively. In addition, we assume that $\boldsymbol{L}=\left(L^{1}, L^{2}\right)$ are independent of $\mathcal{F}_{T}$. We note that the standard investor has the knowledge on the (marginal and joint) laws of $\boldsymbol{L}$ while the insider knows the realization of these thresholds at the renewal times of information. Let the law of $\boldsymbol{L}$ be given by a Gumbel-Barnett copula (see [13]) with parameter $0 \leq \theta \leq 1$, which is given by

$$
C\left(u_{1}, u_{2}\right)=u_{1}+u_{2}-1+\left(1-u_{1}\right)\left(1-u_{2}\right) e^{-\theta \ln \left(1-u_{1}\right) \ln \left(1-u_{2}\right)}
$$

Then the joint cumulative distribution function of $\left(L^{1}, L^{2}\right)$ is given by

$$
F\left(x_{1}, x_{2}\right)=1-e^{-\lambda_{1} x_{1}}-e^{-\lambda_{2} x_{2}}+e^{-\left(\lambda_{1} x_{1}+\lambda_{2} x_{2}+\theta \lambda_{1} \lambda_{2} x_{1} x_{2}\right)}
$$

Moreover, by (4.24), one has

$$
\frac{1}{\zeta\left(x_{1}, x_{2}\right)}=e^{-\left(\theta \lambda_{1} \lambda_{2} x_{1} x_{2}\right)}\left(\left(\theta \lambda_{1} x_{1}+1\right)\left(\theta \lambda_{2} x_{2}+1\right)-\theta\right)
$$

Let denote $\nu=\mu-\frac{\sigma^{2}}{2}$. We recall that for a geometric Brownian motion $X$ with drift $\mu$ and volatility $\sigma$ starting from $X_{0}=1$, the density of the couple $\left(X_{t}^{*}, X_{t}\right)$ for $t>0$ is given by

$$
f_{t}(u, v)=1_{\{u \leq v\}} 1_{\{0 \leq u \leq 1\}} \frac{2 v^{\nu / \sigma^{2}-1} \ln \left(v / u^{2}\right)}{\sigma^{3} \sqrt{2 \pi} t^{(3 / 2)} u} e^{-\frac{\nu^{2} t}{2 \sigma^{2}}} e^{-\frac{\ln ^{2}\left(v / u^{2}\right)}{2 \sigma^{2} t}}
$$

and the density of $X_{t}^{*}$ is given by

$f_{X_{t}^{*}}(w)=1_{\{0<w \leq 1\}}\left(\frac{1}{\sqrt{2 \pi t} w}\left(e^{-\frac{(-\ln (w)+\nu t)^{2}}{2 \sigma^{2} t}}+w^{2 \nu / \sigma^{2}} e^{-\frac{(-\ln (w)-\nu t)^{2}}{2 \sigma^{2} t}}\right)-\frac{\nu}{\sigma^{2}} w^{2 \nu / \sigma^{2}-1} \operatorname{Erfc}\left(\frac{-\ln (w)-\nu t}{\sigma \sqrt{2 t}}\right)\right)$.

where $\operatorname{Erfc}(x)=\frac{2}{\sqrt{\pi}} \int_{x}^{+\infty} e^{-v^{2}} d v, x \geq 0$.

We now present the explicit formulas for the conditional survival probabilities as below.

\subsubsection{Survival probability for $t \in\left[t_{1}, t_{2}\right)$}

\section{Insider information}

$$
\mathbb{P}\left(\tau>T \mid \mathcal{G}_{t}^{I}\right)=1_{\{\tau>t\}} \int_{0}^{+\infty} \mathbb{E}^{\mathbb{P}}\left[1_{\left\{X_{\left[t, t_{2}[\right.}^{*}>x^{1}\right\}} 1_{\left\{X_{\left[t_{2}, T\right.}^{*}>y\right\}} \mid \mathcal{F}_{t}\right]_{x^{1}=L^{1}} \frac{1}{\zeta\left(L^{1}, y\right)} \lambda_{2} e^{-\lambda_{2} y} d y
$$


since $\int_{0}^{+\infty} \frac{1}{\zeta\left(L^{1}, y\right)} \lambda_{2} e^{-\lambda_{2} y} d y=1$. To compute more explicitly this quantity, we need the joint law of the running minimum $\left(X_{\left[t, t_{2}[\right.}^{*}, X_{\left[t_{2}, s\right]}^{*}\right)$. Using the result of [6], one has

$$
\begin{aligned}
\mathbb{E}^{\mathbb{P}}\left[1_{\left\{X_{t_{2}, T[}^{*}>y\right\}} \mid \mathcal{F}_{t_{2}}\right] & =1_{\left\{y \leq X_{t_{2}}\right\}}\left(1-\frac{1}{2} \operatorname{Erfc}\left(\frac{\ln \left(X_{t_{2}} / y\right)+\nu\left(T-t_{2}\right)}{\sigma \sqrt{2\left(s-t_{2}\right)}}\right)\right) . \\
& -\frac{1}{2}\left(\frac{y}{X_{t_{2}}}\right)^{2 \nu / \sigma^{2}} \operatorname{Erfc}\left(\frac{\ln \left(X_{t_{2}} / y\right)-\nu\left(T-t_{2}\right)}{\sigma \sqrt{2\left(T-t_{2}\right)}}\right) \\
& =: G\left(X_{t_{2}}, y\right) .
\end{aligned}
$$

Futhermore, using the markov property and the joint law of $\left(X_{t_{2}-t}^{*}, X_{t_{2}-t}\right)$, it leads to

$$
\mathbb{E}^{\mathbb{P}}\left[1_{\left\{X_{\left[t, t_{2} \mid\right.}^{*}>x^{1}\right\}} 1_{\left\{X_{\left[t_{2}, T\right]}^{*}>y\right\}} \mid \mathcal{F}_{t}\right]=\iint 1_{\left\{u X_{t}>x^{1}\right\}} G\left(v X_{t}, y\right) f_{t_{2}-t}(u, v) d u d v
$$

Standard information For the progressive information, we use $(5.3)$ where successive conditioning implies that

$$
\begin{aligned}
& \mathbb{P}\left(\tau>T \mid \mathcal{F}_{t}\right)=\int_{0}^{1} \int_{0}^{+\infty} \int_{0}^{1} e^{-\lambda_{1} \min \left(X_{t}^{*}, u X_{t}\right)-\lambda_{2}\left(v w X_{t}\right)-\theta \lambda_{1} \lambda_{2} \min \left(X_{t}^{*}, u X_{t}\right)\left(v w X_{t}\right)} \\
& f_{X_{T-t_{2}}^{*}}(w) f_{t_{2}-t}(u, v) d w d v d u
\end{aligned}
$$

\subsubsection{Survival probability for $t \in\left[t_{2}, T\right)$}

Straightforward computations imply the following results.

\section{Insider information}

$$
\mathbb{P}\left(\tau>T \mid \mathcal{G}_{t}^{I}\right)=\left.1_{\{\tau>t\}} \int_{u}^{1} f_{X_{T-t}^{*}}(w) d w\right|_{u=\frac{L 1}{X_{t}}}
$$

\section{Standard information}

$$
\mathbb{P}\left(\tau>T \mid \mathcal{G}_{t}\right)=1_{\{\tau>t\}} \frac{\int_{0}^{1} F\left(X_{t_{2}}^{*}, \min \left(X_{\left[t_{2}, t\right.}^{*}, w X_{t}\right)\right) f_{X_{T-t}^{*}}(w) d w}{F\left(X_{t_{2}}^{*}, X_{\left[t_{2}, t[\right.}^{*}\right)}
$$

\section{$5.3 \quad$ Numerical results}

In this subsection, we compare the survival probabilities for insider and standard investor by numerical examples. We use the default time model described previously. The value of the parameters are $\mu=0.05, \sigma=0.8, \lambda_{1}=1.5$ and $\lambda_{2}=1, t_{1}=0, t_{2}=1$ and $t_{3}=T=2$. In particular, we analyse the impact of the correlation between $L^{1}$ and $L^{2}$ through the parameter 
$\theta$. The case $\theta=0$ corresponds to the independence case. We present two examples. In the first one, there is a default event before the maturity and in the second one, there is no default. In each example, we compare the survival probabilities $P\left(\tau>T \mid \mathcal{G}_{t}^{I}\right)$ and $P\left(\tau>T \mid \mathcal{G}_{t}\right)$ on a given trajectory of the firm value.

In the first example, Figure 1 presents the realized trajectory of the firm value. We suppose that the manager adjust the threshold level at $t_{2}=1$ from $L^{1}=0.8$ to $L^{2}=1.5$, so there is a high risk of default after time $t_{2}$, which is larger than the expected value. We observe from the three graphs in Figure 2 that in all the cases (for different values of $\theta$ ), the insider will modify immediately the estimations on the survival probability and there is an instantaneous jump at $t_{2}$. While the standard investor, who is not accessible to this information, maintain the survival probability at a high level and can adjust the estimation only when the default occurs effectively. Finally comparing the three graphs where the correlation between $L^{1}$ and $L^{2}$ varies, we see that when the time approaches $t_{2}$, since the firm value is at a relatively high level compared to $L^{1}$, when there is a strong correlation (with larger $\theta$ ) between the two thresholds, the insider will have a higher estimation for the survival probability than when there is independence. However, such difference between the estimations which are due to different values of $\theta$ will be neutralized once the insider get the exact information on $L^{2}$ at time $t_{2}$.

Figure 1: First Case : Default during $[1,2], L^{1}=0.8, L^{2}=1.5$

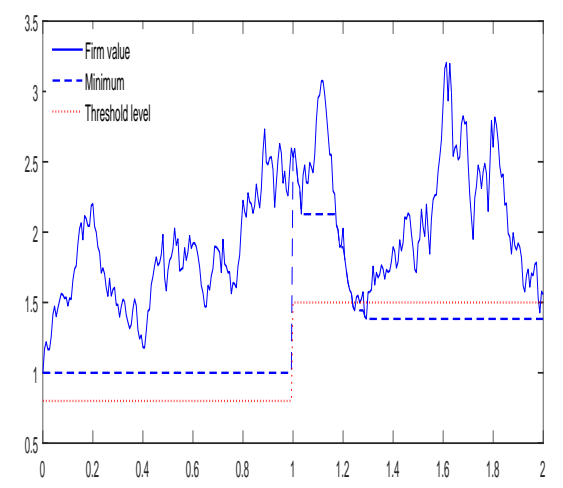

In the second example where the sample path of the firm value is given by Figure 3 , there is no default before the maturity $T$. In addition, we suppose that the level of the second threshold $L^{2}=0.6$ is slightly lower than the first one $L^{1}=0.8$ and is close to the expected value. So there is no important readjustment of the insider's estimation at $t_{2}$, as shown by all the three graphs in Figure 4. However, when the firm value descends gradually after time $t_{2}$ and approaches the threshold level $L^{2}$, the estimations of the survival probability by the insider has dropped significantly. Only when the firm value begins to go up back and when the time approaches the maturity, the insider modifies once again the survival probability to be higher. In contrast, the estimations by the standard investor remain quite stable during all the period in this example. The comparison between the correlation parameter $\theta$ is similar as in the first example. Since the firm value is at a high level during the first period, if $\theta=1$, the insider has a higher estimation for 
Figure 2: Survival Probabilities $P\left(\tau>T \mid \mathcal{G}_{t}^{I}\right)$ and $P\left(\tau>T \mid \mathcal{G}_{t}\right)$ for $\theta=0,0.5$ and 1
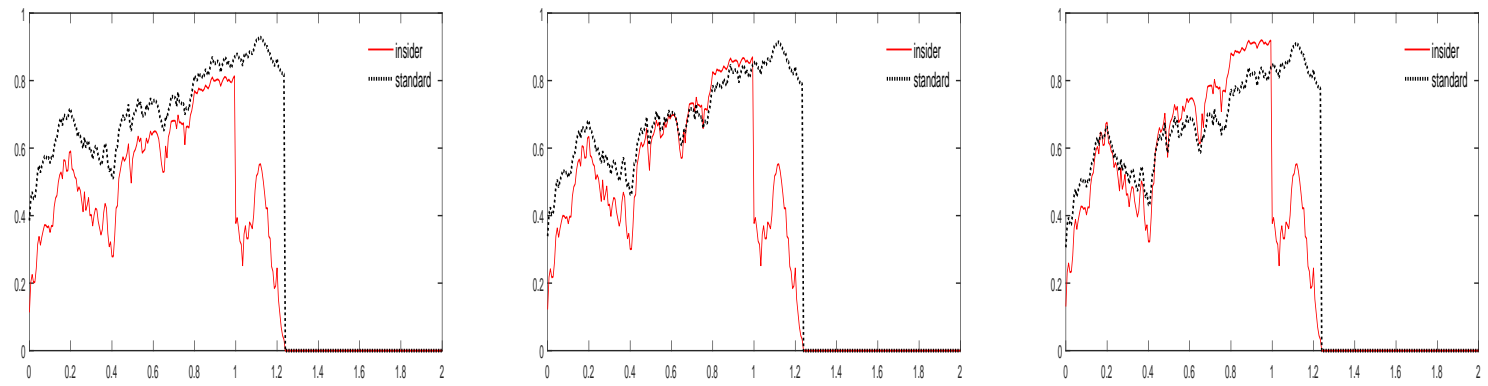

the survival probability than in the case if $\theta=0$. However, such differences are visible only before the second information renewal time.

Figure 3: Second Case : No Default, $L^{1}=0.8, L^{2}=0.6$

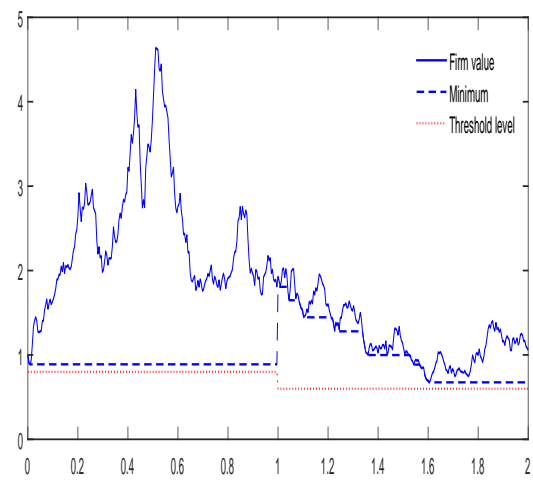

\section{Appendix}

\subsection{Proof of Proposition 4.1}

The goal of this subsection is to apply Theorem 3.13 to compute $\mathbb{G}^{I}$-conditional expectations under Assumption 2. We begin by calculating, in several lemmas below, the recursive operators in Theorem 3.13 in an explicit manner and then give the proof of Proposition 4.1. Throughout this Subsection 6.1 Assumption 2 holds.

Lemma 6.1 Let $i \in\{1, \ldots, n\}$ and $t \in\left[t_{i}, T\right]$. If $X_{t}\left(\boldsymbol{L}^{(i)}\right)$ is a non-negative $\mathcal{G}_{t}^{i}$-measurable random 
Figure 4: Survival Probabilities $P\left(\tau>T \mid \mathcal{G}_{t}^{I}\right)$ and $P\left(\tau>T \mid \mathcal{G}_{t}\right)$ for $\theta=0,0.5$ and 1
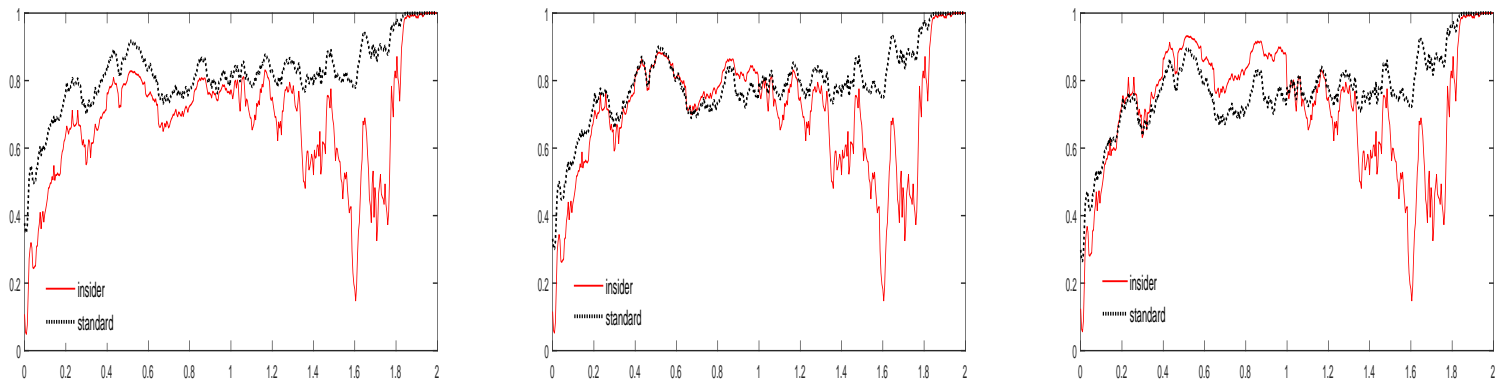

variable. Then one has

$$
J_{i}\left(X_{t}\left(\boldsymbol{L}^{(i)}\right)\right)=\left.\int_{E} \frac{\mathbb{E}^{\mathbb{P}}\left[X_{t}\left(\boldsymbol{x}^{(i)}\right) Z_{t}^{i-1}\left(\boldsymbol{x}^{(i-1)}\right) \mid \mathcal{F}_{t_{i}}\right]}{Z_{t_{i}}^{i-1}\left(\boldsymbol{x}^{(i-1)}\right)}\right|_{\boldsymbol{x}^{(i-1)}=\boldsymbol{L}^{(i-1)}} \beta_{t_{i}}^{i \mid i-1}\left(\boldsymbol{L}^{(i-1)}, x^{i}\right) \mathbb{P}\left(L^{i} \in d x^{i} \mid \mathcal{G}_{0}^{i-1}\right) .
$$

where $Z_{t}^{i}\left(\boldsymbol{x}^{(i)}\right)$ is defined by 4.5 .

Proof: We recall the operation $J_{i}$ defined by (3.17). By (3.18) one has

$$
J_{i}\left(X_{t}\left(\boldsymbol{L}^{(i)}\right)\right)=\left.\int_{E} \frac{\mathbb{E}^{\mathbb{P}}\left[X_{t}\left(\boldsymbol{x}^{(i)}\right) \psi_{t}^{i-1}\left(\boldsymbol{x}^{(i-1)}\right)^{-1}\right]}{\psi_{t_{i}}^{i-1}\left(\boldsymbol{x}^{(i-1)}\right)^{-1}}\right|_{\boldsymbol{x}^{(i-1)}=\boldsymbol{L}^{(i-1)}} \mathbb{P}\left(L^{i} \in d x^{i} \mid \mathcal{G}_{t_{i}}^{i-1}\right)
$$

Note that

$$
\psi_{t}^{i-1}\left(\boldsymbol{x}^{(i-1)}\right)^{-1}=\prod_{k=1}^{i-1} \alpha_{t}^{k \mid k-1}\left(\boldsymbol{x}^{(k)}\right)=\prod_{k=1}^{i-1} \frac{\beta_{t}^{k \mid k-1}\left(\boldsymbol{x}^{(k)}\right)}{\beta_{t_{k} \mid k-1}^{k\left(\boldsymbol{x}^{(k)}\right)}}=Z_{t}^{i-1}\left(\boldsymbol{x}^{(i-1)}\right) \prod_{k=1}^{i-1} \frac{1}{\beta_{t_{k}}^{k \mid k-1}\left(\boldsymbol{x}^{(k)}\right)},
$$

where the first equality comes from (3.11), and the second equality follows from 4.4), and the last equality results from 4.5. Similarly, one has

$$
\psi_{t_{i}}^{i-1}\left(\boldsymbol{x}^{(i-1)}\right)^{-1}=\prod_{k=1}^{i-1} \frac{\beta_{t_{i}}^{k \mid k-1}\left(\boldsymbol{x}^{(k)}\right)}{\beta_{t_{k}}^{k \mid k-1}\left(\boldsymbol{x}^{(k)}\right)}=Z_{t_{i}}^{i-1}\left(\boldsymbol{x}^{(i-1)}\right) \prod_{k=1}^{i-1} \frac{1}{\beta_{t_{k}}^{k \mid k-1}\left(\boldsymbol{x}^{(k)}\right)} .
$$

Therefore

$$
\frac{\mathbb{E}^{\mathbb{P}}\left[X_{t}\left(\boldsymbol{x}^{(i)}\right) \psi_{t}^{i-1}\left(\boldsymbol{x}^{(i-1)}\right)^{-1}\right]}{\psi_{t_{i}}^{i-1}\left(\boldsymbol{x}^{(i-1)}\right)^{-1}}=\frac{\mathbb{E}^{\mathbb{P}}\left[X_{t}\left(\boldsymbol{x}^{(i)}\right) Z_{t}^{i-1}\left(\boldsymbol{x}^{(i-1)}\right) \mid \mathcal{F}_{t_{i}}\right]}{Z_{t_{i}}^{i-1}\left(\boldsymbol{x}^{(i-1)}\right)} .
$$

By (4.2), we obtain the announced equality.

Lemma 6.2 The pricing kernel (3.25) is given, under Assumption 2, by

$$
\Phi_{t_{i+2}}\left(\boldsymbol{L}^{(i+1)}\right)=\frac{\beta_{t_{i+2}^{i+1 \mid i}}^{i\left(\boldsymbol{L}^{(i+1)}\right)}}{\beta_{t_{i+1}}^{i+1 \mid i}\left(\boldsymbol{L}^{(i+1)}\right)}
$$


and

$$
J_{i+1}\left(\Phi_{t_{i+2}}\left(\boldsymbol{L}^{(i+1)}\right)\right)=1 .
$$

Proof: One has

$$
\Phi_{t_{i+2}}\left(\boldsymbol{L}^{(i+1)}\right)=\left(J_{i+2} \circ \cdots \circ J_{n}\right)\left(\alpha_{t_{i+2}}^{i+1 \mid i}\left(\boldsymbol{L}^{(i)}\right) \cdots \alpha_{T}^{n \mid n-1}\left(\boldsymbol{L}^{(n)}\right)\right) .
$$

By Lemma 6.1, one can express it as the integral of

$$
\begin{aligned}
& \mathbb{E}^{\mathbb{P}}\left[\alpha_{t_{i+2}}^{i+1 \mid i}\left(\boldsymbol{x}^{(i+1)}\right) \prod_{j=i+2}^{n}\left(\alpha_{t_{j+1}}^{j \mid j-1}\left(\boldsymbol{x}^{(j)}\right) \frac{Z_{t_{j+1}}^{j-1}\left(\boldsymbol{x}^{(j-1)}\right)}{Z_{t_{j}}^{j-1}\left(\boldsymbol{x}^{(j-1)}\right)} \beta_{t_{j}}^{j \mid j-1}\left(\boldsymbol{x}^{(j)}\right)\right) \mid \mathcal{F}_{t_{i+2}}\right]_{\boldsymbol{x}^{(i+1)}=\boldsymbol{L}^{(i+1)}} \\
& =\frac{\beta_{t_{i+2}}^{i+1 \mid i}\left(\boldsymbol{L}^{(i+1)}\right)}{\beta_{t_{i+1}}^{i+1 \mid i}\left(\boldsymbol{L}^{(i+1)}\right)} \cdot \frac{\mathbb{E}\left[Z_{T}^{n}(\boldsymbol{x}) \mid \mathcal{F}_{t_{i+2}}\right]_{\boldsymbol{x}^{(i+1)}=\boldsymbol{L}^{(i+1)}}}{Z_{t_{i+2}}^{i+1}\left(\boldsymbol{L}^{(i+1)}, x^{i+2}\right)}=\frac{\beta_{t_{i+2}}^{i+1 \mid i}\left(\boldsymbol{L}^{(i+1)}\right)}{\beta_{t_{i+1}}^{i+1 \mid i}\left(\boldsymbol{L}^{(i+1)}\right)} \cdot \frac{Z_{t_{i+2}}^{n}\left(\boldsymbol{L}^{(i+1)}, x^{i+2}, \cdots, x^{n}\right)}{Z_{t_{i+2}}^{i+1}\left(\boldsymbol{x}^{(i+2)}\right)} .
\end{aligned}
$$

with respect to $\mathbb{P}\left(L^{i+2} \in d x^{i+2}, \cdots, L^{n} \in d x^{n} \mid \mathcal{G}_{0}^{i+1}\right)$. By (4.7) we obtain the first equality. We then apply Lemma 6.1 to write $J_{i+1}\left(\Phi_{t_{i+2}}\left(\boldsymbol{L}^{(i+1)}\right)\right)$ as

$$
\begin{aligned}
& \int_{E} \mathbb{E}^{\mathbb{P}}\left[\frac{\beta_{t_{i+2}}^{i+1 \mid i}\left(\boldsymbol{x}^{(i+1)}\right)}{\beta_{t_{i+1}}^{i+1 \mid i}\left(\boldsymbol{x}^{(i+1)}\right)} \cdot \frac{Z_{t_{i+2}}^{i}\left(\boldsymbol{x}^{(i)}\right)}{Z_{t_{i+1}}^{i}\left(\boldsymbol{x}^{(i)}\right)} \beta_{t_{i+1}}^{i+1 \mid i}\left(\boldsymbol{x}^{(i+1)}\right) \mid \mathcal{F}_{t_{i+1}}\right]_{\boldsymbol{x}^{(i)}=\boldsymbol{L}^{(i)}} \mathbb{P}\left(L^{i+1} \in d x^{i+1} \mid \mathcal{G}_{0}^{i}\right) \\
= & \int_{E} \mathbb{E}^{\mathbb{P}}\left[\frac{Z_{t_{i+2}}^{i}\left(\boldsymbol{x}^{(i)}\right)}{Z_{t_{i+1}}^{i}\left(\boldsymbol{x}^{(i)}\right)} \beta_{t_{i+2}}^{i+1 \mid i}\left(\boldsymbol{x}^{(i+1)}\right) \mid \mathcal{F}_{t_{i+1}}\right]_{\boldsymbol{x}^{(i)}=\boldsymbol{L}^{(i)}} \mathbb{P}\left(L^{i+1} \in d x^{i+1} \mid \mathcal{G}_{0}^{i}\right) \\
= & \int_{E} \mathbb{E}^{\mathbb{P}}\left[\frac{Z_{t_{i+2}}^{i+1}\left(\boldsymbol{x}^{(i+1)}\right)}{Z_{t_{i+1}}^{i}\left(\boldsymbol{x}^{(i)}\right)} \mid \mathcal{F}_{t_{i+1}}\right]_{\boldsymbol{x}^{(i)}=\boldsymbol{L}^{(i)}} \mathbb{P}\left(L^{i+1} \in d x^{i+1} \mid \mathcal{G}_{0}^{i}\right) .
\end{aligned}
$$

Note that by Lemma 4.2 one has

$$
\mathbb{P}\left(L^{1} \in d x^{1}, \cdots, L^{i} \in d x^{i} \mid \mathcal{F}_{t}\right)=Z_{t}^{i}\left(\boldsymbol{x}^{(i)}\right) \mathbb{P}\left(L^{1} \in d x^{1}, \cdots, L^{i} \in d x^{i} \mid \mathcal{F}_{0}\right)
$$

Therefore $\left(Z_{t}^{i+1}\left(\boldsymbol{x}^{(i+1)}\right)\right)_{t \in[0, T]}$ is a $(\mathbb{F}, \mathbb{P})$-martingale, so we obtain

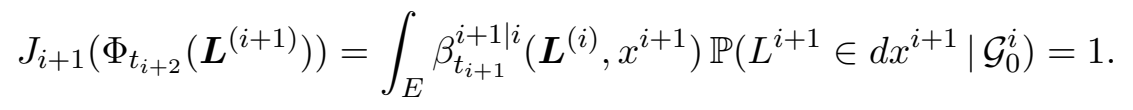

Proof of Proposition 4.1 Let $Y_{T}(\boldsymbol{L})$ be a non-negative $\mathcal{G}_{T}^{n}$-measurable random variable. Then for $t \in[0, T]$ one has $\mathbb{E}^{\mathbb{P}}\left[Y_{T}(\boldsymbol{L}) \mid \mathcal{G}_{t}^{I}\right]=\left.\sum_{i=1}^{n} 1_{\left[t_{i}, t_{i+1}\right)}(t) \int_{E^{n-i}} \frac{\mathbb{E}^{\mathbb{P}}\left[Y_{T}(\boldsymbol{x}) Z_{T}^{n}(\boldsymbol{x}) \mid \mathcal{F}_{t}\right]}{Z_{t}^{i}\left(\boldsymbol{x}^{(i)}\right)}\right|_{\boldsymbol{x}^{(i)}=\boldsymbol{L}^{(i)}} \mathbb{P}\left(L^{i+1} \in d x^{i+1}, \cdots, L^{n} \in d x^{n} \mid \mathcal{G}_{0}^{i}\right)$. 
Proof: We apply Theorem 3.13 and compute the sequence of random variables $\left(Y_{t_{i+1}}\left(\boldsymbol{L}^{(i)}\right)\right)_{i=0}^{n}$ under Assumption 2. By the backward recursive relation (3.24) and the equalities (6.2) and (6.3), one has

$$
Y_{t_{i+1}}\left(\boldsymbol{L}^{(i)}\right)=\frac{J_{i+1}\left(Y_{t_{i+2}}\left(\boldsymbol{L}^{(i+1)}\right) \Phi_{t_{i+2}}\left(\boldsymbol{L}^{(i+1)}\right)\right)}{J_{i+1}\left(\Phi_{t_{i+2}}\left(\boldsymbol{L}^{(i+1)}\right)\right)}=J_{i+1}\left(Y_{t_{i+2}}\left(\boldsymbol{L}^{(i+1)}\right) \frac{\beta_{t_{i+2}}^{i+1 \mid i}\left(\boldsymbol{L}^{(i+1)}\right)}{\beta_{t_{i+1}}^{i+1 \mid i}\left(\boldsymbol{L}^{(i+1)}\right)}\right),
$$

where the second equality comes from 3.20. By Lemma 6.1. we can write it as

$$
\begin{aligned}
& \int_{E} \mathbb{E}^{\mathbb{P}}\left[Y_{t_{i+2}}\left(\boldsymbol{x}^{(i+1)}\right) \frac{\beta_{t_{i+2}}^{i+1 \mid i}\left(\boldsymbol{x}^{(i+1)}\right)}{\beta_{t_{i+1}}^{i+1 \mid i}\left(\boldsymbol{x}^{(i+1)}\right)} \cdot \frac{Z_{t_{i+2}}^{i}\left(\boldsymbol{x}^{(i)}\right)}{Z_{t_{i+1}}^{i}\left(\boldsymbol{x}^{(i)}\right)} \beta_{t_{i+1}}^{i+1 \mid i}\left(\boldsymbol{x}^{(i+1)}\right) \mid \mathcal{F}_{t_{i+1}}\right]_{\boldsymbol{x}^{(i)}=\boldsymbol{L}^{(i)}} \mathbb{P}\left(L^{i+1} \in d x^{i+1} \mid \mathcal{G}_{0}^{i}\right) \\
= & \int_{E} \mathbb{E}^{\mathbb{P}}\left[Y_{t_{i+2}}\left(\boldsymbol{x}^{(i+1)}\right) \frac{Z_{t_{i+2}}^{i}\left(\boldsymbol{x}^{(i)}\right)}{Z_{t_{i+1}}^{i}\left(\boldsymbol{x}^{(i)}\right)} \beta_{t_{i+2}}^{i+1 \mid i}\left(\boldsymbol{x}^{(i+1)}\right) \mid \mathcal{F}_{t_{i+1}}\right]_{\boldsymbol{x}^{(i)}=\boldsymbol{L}^{(i)}} \mathbb{P}\left(L^{i+1} \in d x^{i+1} \mid \mathcal{G}_{0}^{i}\right) .
\end{aligned}
$$

Therefore we obtain that $Y_{t_{i+1}}\left(\boldsymbol{L}^{(i)}\right)$ is the integral

$$
\begin{aligned}
& \int_{E^{n-i}} \mathbb{E}^{\mathbb{P}}\left[Y_{T}(\boldsymbol{x}) \prod_{j=i+1}^{n} \frac{Z_{t_{j+1}-1}^{j-1}\left(\boldsymbol{x}^{(j-1)}\right)}{Z_{t_{j}}^{j-1}\left(\boldsymbol{x}^{(j-1)}\right)} \beta_{t_{j+1}^{j \mid j-1}}\left(\boldsymbol{x}^{(j)}\right) \mid \mathcal{F}_{t_{i+1}}\right]_{\boldsymbol{x}^{(i)}=\boldsymbol{L}^{(i)}} \mathbb{P}\left(L^{n} \in d x^{n} \mid \mathcal{G}_{0}^{n-1}\right) \cdots \mathbb{P}\left(L^{i+1} \in d x^{i+1} \mid \mathcal{G}_{0}^{i}\right) \\
& =\left.\int_{E^{n-i}} \frac{\mathbb{E}^{\mathbb{P}}\left[Y_{T}(\boldsymbol{x}) Z_{T}^{n}(\boldsymbol{x}) \mid \mathcal{F}_{t_{i+1}}\right]}{Z_{t_{i+1}}^{i}\left(\boldsymbol{x}^{(i)}\right)}\right|_{\boldsymbol{x}^{(i)}=\boldsymbol{L}^{(i)}} \mathbb{P}\left(L^{i+1} \in d x^{i+1}, \cdots, L^{n} \in d x^{n} \mid \mathcal{G}_{0}^{i}\right)
\end{aligned}
$$

We deduce that, for $t \in\left[t_{i}, t_{i+1}\right)$ one has

$$
\begin{aligned}
& \left.\frac{\mathbb{E}^{\mathbb{P}}\left[Y_{t_{i+1}}\left(\boldsymbol{x}^{(i)}\right) \psi_{t_{i+1}}\left(\boldsymbol{x}^{(i)}\right)^{-1} \mid \mathcal{F}_{t}\right]}{\psi_{t}^{i}\left(\boldsymbol{x}^{(i)}\right)^{-1}}\right|_{\boldsymbol{x}^{(i)}=\boldsymbol{L}^{(i)}} \\
= & \left.\int_{E^{n-i}} \frac{\mathbb{E}^{\mathbb{P}}\left[Y_{T}(\boldsymbol{x}) Z_{T}^{n}(\boldsymbol{x}) \mid \mathcal{F}_{t}\right]}{Z_{t}^{i}\left(\boldsymbol{x}^{(i)}\right)}\right|_{\boldsymbol{x}^{(i)}=\boldsymbol{L}^{(i)}} \mathbb{P}\left(L^{i+1} \in d x^{i+1}, \cdots, L^{n} \in d x^{n} \mid \mathcal{G}_{0}^{i}\right) .
\end{aligned}
$$

The proposition is thus proved.

\section{References}

[1] J. Amendinger. Martingale representation theorems for initially enlarged filtrations. Stochastic Processes and their Applications, 89(1):101 - 116, 2000.

[2] J. Amendinger, P. Imkeller, and M. Schweizer. Additional logarithmic utility of an insider. Stochastic Processes and their Applications, 75(2):263 - 286, 1998.

[3] F. Biagini and B. Øksendal. A general stochastic calculus approach to insider trading. Applied Mathematics and Optimization, 52(2):167-181, 2005. 
[4] T. R. Bielecki, M. Jeanblanc, and M. Rutkowski. Modeling and valuation of credit risk. In Stochastic methods in finance, volume 1856 of Lecture Notes in Math., pages 27-126. Springer, Berlin, 2004.

[5] T. R. Bielecki and M. Rutkowski. Credit Risk : modeling, valuation and hedging. Springer, Berlin, 2002.

[6] A. Borodin and P. Salminen. Handbook of Brownian Motion: Facts and Formulae, volume 1873 of Probability and Its Applications. Birkhauser Basel, 1996.

[7] J. M. Corcuera, P. Imkeller, A. Kohatsu-Higa, and D. Nualart. Additional utility of insiders with imperfect dynamical information. Finance and Stochastics, 8(3):437-450, 2004.

[8] C. Dellacherie and P.A. Meyer. Probabilités et Potentiel, Espaces Mesurables Tome 1. Enseignement des sciences. Hermann, 2008.

[9] N. El Karoui, M. Jeanblanc, and Y. Jiao. Dynamics of multivariate default system in random environment. preprint hal-01205753, 2015.

[10] R. J. Elliott, M. Jeanblanc, and M. Yor. On models of default risk. Mathematical Finance, 10(2):179-195, 2000.

[11] H. Föllmer and P. Imkeller. Anticipation cancelled by a Girsanov transformation: a paradox on Wiener space. Annales de l'Institut Henri Poincaré. Probabilités et Statistiques, 29(4):569$586,1993$.

[12] A. Grorud and M. Pontier. Insider trading in a continuous time market model. International Journal of Theoretical and Applied Finance, 1(3):331-347, 1998.

[13] E. J. Gumbel. Bivariate logistic distributions. Journal of the American Statistical Association, 56(294):335-349, 1961.

[14] C. Hillairet and Y. Jiao. Credit risk with asymmetric information on the default threshold. Stochastics, 84(2-3):183-198, 2012.

[15] P. Imkeller. Random times at which insiders can have free lunches. Stochastics, 74(1-2):465487, 2002.

[16] P. Imkeller. Malliavin's calculus in insider models: Additional utility and free lunches. Mathematical Finance, 13(1):153-169, 2003.

[17] J. Jacod. Calcul stochastique et problèmes de martingales, volume 714 of Lecture Notes in Math. Springer, Berlin, 1979.

[18] J. Jacod. Grossissement initial, hypothèse H' et théorème de Girsanov. In Séminaire de calcul stochastique 1982 - 83, Paris, volume 1118 of Lecture Notes in Math. Springer-Verlag, Berlin, 1985 .

[19] T. Jeulin. Semi-martingales et grossissement d'une filtration, volume 833 of Lecture Notes in Mathematics. Springer, Berlin, 1980. 
[20] T. Jeulin and M. Yor. Grossissement d'une filtration et semi-martingales: formules explicites. In Séminaire de Probabilités, XII (Univ. Strasbourg, Strasbourg, 1976/1977), volume 649 of Lecture Notes in Math., pages 78-97. Springer, Berlin, 1978.

[21] T. Jeulin and M. Yor, editors. Grossissements de filtrations: exemples et applications, volume 1118 of Lecture Notes in Mathematics. Springer-Verlag, Berlin, 1985. Papers from the seminar on stochastic calculus held at the Université de Paris VI, Paris, 1982/1983.

[22] Y. Kchia, M. Larsson, and P. Protter. Linking progressive and initial filtration expansions. In F. Viens, J. Feng, Y. Hu, and E. Nualart, editors, Malliavin Calculus and Stochastic Analysis, volume 34 of Springer Proceedings in Mathematics 8 Statistics, pages 469-487. Springer US, 2013.

[23] Y. Kchia and P. Protter. On progressive filtration expansions with a process; applications to insider trading. International Journal of Theoretical and Applied Finance 03/2014; 18(04)., 2014.

[24] J. Mansuy and M. Yor. Random Times and Enlargements of Filtrations in a Brownian Setting, volume 1873 of Lecture Notes in Math. Springer-Verlag, Berlin, 2006.

[25] P.A. Meyer. Une remarque sur le calcul stochastique dépendant d'un paramètre. In C. Dellacherie, P.A. Meyer, and M. Weil, editors, Séminaire de Probabilités XIII, volume 721 of Lecture Notes in Mathematics, pages 199-215. Springer Berlin Heidelberg, 1979.

[26] S. Song. Grossissement de filtrations et problèmes connexes. PhD thesis, Université Paris VII, 1987.

[27] S. Song. Local solution method for the problem of enlargement of filtration. ArXiv e-prints 1302.2862, February 2013. 